\title{
Continuing Wars of Attrition
}

\author{
by \\ R. Preston McAfee* \\ University of Texas
}

\begin{abstract}
This paper presents a new model of the war of attrition, based on a geographical or territorial dispute. Both sides try to extend their boundaries, and the level of effort at each time is endogenous. There are two kinds of stationary equilibria, one with fighting to completion, the other with a cessation of hostilities. As a player gets closer to losing, that player's probability of winning battles falls, social welfare rises, and the levels of effort of both players rise. A draw or standoff is possible. The theory is applied to a variety of conflicts, including wars and attempts at market domination.
\end{abstract}

* Department of Economics, University of Texas, Austin, Texas, 78712; mcafee@eco.utexas.edu. Scott Freeman, John McMillan, and John Morgan have provided useful comments. 


\section{Introduction}

"The guerrilla wins if he does not lose." -Henry Kissinger ${ }^{1}$

It is generally believed that a single browser, made either by Netscape or Microsoft, will come to dominate the market for internet browsers. The competition between the two companies is sufficiently fierce that both have given their browsers to consumers without charge. This war of attrition makes sense provided each company stands a significant chance of winning the war. The contestants, in this instance, are decidedly asymmetric. Microsoft, with its enormous profits on operating systems, can sustain losses that would bankrupt Netscape. Netscape is, essentially, fighting for its life; a loss in the browser market would turn Netscape into a website. In this fight, Netscape has a low opportunity cost of continuing the fight, since it will be obliterated by a loss in the browser market. Microsoft, in contrast, bears a high opportunity cost of the fight, in that resources invested in browsers could be invested in other high return activities like paying shareholders, but Microsoft also has a very large budget. The contest is decidedly asymmetric.

Amazon dominates the market for internet-based book sales. Barnes and Noble, a large conventional bookseller, is Amazon's major challenger for this currently unprofitable market. Once again, we have a company with one single business being challenged by a company with another, unchallenged core business, although in this case, the market may support two or more competing sellers. Unlike the case of Microsoft, Amazon is sometimes thought to be the "deep pockets" firm, because it is investing money raised in the stock market, while Barnes and Noble is investing profits from its core business. As a consequence, Barnes and Noble has sold part of its internet company, to raise additional capital. It may be that the market can support both firms, but the competition between these two firms, which has involved massive investments in the websites and a great deal of litigation, appears to be similar to the competition between Microsoft and Netscape.

Asymmetries in the abilities of contestants to survive a protracted competition appear to be more common than symmetric competitions. The US involvement in Vietnam, which is often described as a war of attrition, had much the same flavor of the Microsoft/Netscape fight, with the US in the role of Microsoft, having a large budget and a high political cost of a protracted war. (see, e.g. Mack, 1975.) France's experiences in both Vietnam and Algeria have a similar flavor (Paret, 1964, and Mack, 1975), as does the Irish War of Independence. ${ }^{2}$ The Nazis continually expected the Soviets to run out of tanks and troops during 1943, and the Soviets did not. (There seems to be some mystery concerning the source of these

\footnotetext{
${ }^{1}$ Quoted by Andrew Mack, 1975.
} 
armored divisions, according to William Shirer, 1959, at p. 1119.) Finally, during the US Civil War, the Union expected the Confederacy to stop fighting as it ran out of materials, while the Confederacy expected that the Union could not sustain its losses politically. The US Civil War appears analogous to the US/Vietnam War and the browser war. ${ }^{3}$ The Union had a high cost of protracted conflict but a much larger budget. The Confederacy perceived itself fighting for its existence, but had very limited resources.

In the early days of US telephony, AT\&T deployed the Long Lines network, and engaged in a series of contests with small, regional phone companies. In the view of some observers, the purpose of these contests was to drive down the stock price of the regional companies, permitting inexpensive acquisition by AT\&T. Whether or not acquisition was AT\&T's intention, the contests were asymmetric; AT\&T could afford to outspend any individual regional company. On the other hand, AT\&T had alternative uses for its resources, while the regional companies might devote the company's entire resources on surviving. ${ }^{4}$

Who should win a war of attrition between very different players? Why doesn't the weaker player concede immediately? The examples provided above have the nature of a war of attrition, in that two sides are competing with each other for a prize that can only accrue to one of them. However, standard models fail to accommodate these examples well. First, all of the contests were decidedly asymmetric -typically with a large player and a smaller player. Second, the competitions involved one firm or country fighting for its existence against a player who can go home in the event of a loss. Typically the latter player is the stronger player in resources. One might view the smaller player as budget constrained, but that doesn't seem to be a good description of Vietnam, Algeria, the Nazi war against the Soviet Union, and the US Civil War, where the budget-constrained small player somehow kept fighting. Third, the level of effort is endogenous -- firms or nations can expend more or less effort at each point in time. The endogenous effort choice is important because even a small player can exert a lot of effort in the near term, perhaps inducing the larger player to exit.

This paper presents a discrete war of attrition. One can think of the game as a football or soccer match along a line segment; a tug of war is the best analogy. ${ }^{5}$ Each player has an end or goal, between which there are a series of nodes at which a battle can occur. The object is push the battle "front" or point of conflict to the other player's end, just as the object in soccer is to get the ball into the other team's goal.

\footnotetext{
${ }^{2}$ Kautt, 1999, explains the Irish victory over a force "superior in technology, industry, military force and population."

${ }^{3}$ See, for example, the statement by Gary Gallagher quoted in Zebrowski, 1999.

${ }^{4}$ See Kaserman and Mayo, 1995. The legal requirement of fiduciary responsibility to the shareholders presents an interesting paradox. By expending resources to survive, the company is driving up the ultimate price AT\&T will pay, increasing value only if a takeover arises, at the same time that the company is expending money that could be paid to shareholders to prevent the profitable takeover.
} 
The first to do so wins, with the other losing. At most one step can be taken in each period; each step may be thought of as a battle. The value of winning is positive, exceeding the value of no resolution (set to zero), and the value of losing is assumed negative.

The main results are (i) that effort tends to rise as either player gets close to winning, (ii) the probability that a player advances rises the closer to winning the player is, (iii) social welfare is u-shaped, with higher utility near the goals. At any point, at least one of the players has negative utility. Moreover, in the central area, the utility of both players may be negative. This does not mean players would unilaterally exit, however, since the negative utility exceeds the utility of an immediate loss. It is possible for the outcome to be a draw, with a weakly stable interior solution. In this case, neither player wins nor loses.

The next section reviews the received wisdom. The third and fourth sections present the theory, and the fifth section revisits applications, discusses when peaceful co-existence is an equilibrium, and concludes.

\section{Extant Theory}

Due perhaps to the origin of the formal theory of the war of attrition in evolutionary biology (Maynard Smith, 1973) and the desire for simplicity, most analyses focus on symmetric games. In all of these analyses, the effort choice is exogenous: firms either stay in, or exit. This paper focuses on asymmetric contests with an endogenous effort choice.

There are two main versions of the war of attrition in the literature. One of these is the first-price all-pay auction, in which each firm chooses an exit point or bid simultaneously and the firm choosing the larger exit point wins, with each paying their bid. ${ }^{6}$ The more popular model is an oral version modeled analogously to Milgrom and Weber's 1982 English auction. ${ }^{7}$ Here the players hold their hands up to signal that they are still in. Exit is irrevocable; when all but one firm exits, the remaining firm wins. ${ }^{8}$

The first price case is often considered as a model of lobbying. In this model, the firms put money in envelopes simultaneously, and give it to a politician. The politician is honest in the sense that he is

\footnotetext{
${ }^{5}$ The model is very closely related to the tug-of-war model of Christopher Harris and John Vickers, 1987.

${ }^{6}$ Baye, Kovenock and de Vries, 1996, Kapur, 1995, Amann and Leininger, 1996, and Krishna and Morgan, 1997.

${ }^{7}$ See Hendricks and Wilson, 1992, 1995 and Hendricks, Weiss and Wilson, 1998.

${ }^{8}$ In an important paper, Jeremy Bulow and Paul Klemperer, 1999, distinguish between the IO version and the standards game of wars of attrition. In the IO version, exit stops one's costs from accruing, while in the standards game, firms continue to incur costs until the penultimate player exits and the game ends. These two are identical in the case of two firms. Moreover, if there is only one winner, in the IO version of the game, Bulow and Klemperer prove that all but two firms drop out immediately, even when the firms are distinguished by privately known costs or values.
} 
bought by the highest bidder, and votes the way this bidder desires. The politician is dishonest in that he keeps all of the bids. Most wars of attrition, and all of the applications discussed in the introduction, take place in real time, and therefore are better modeled in the oral version. Even bribery admits additional contributions.

The all-pay auction is presented in some detail, because it is used as a subgame to the continuing war of attrition. Suppose the prize is $\$ v_{i}$ for each firm $i$. Consider the case of two firms, named 0 and 1 , with $\operatorname{costs} c_{0}(\mathrm{t})$ and $c_{1}(\mathrm{t})$, respectively of remaining in the game up to time t. Costs are strictly increasing. Firm $i$ uses the distribution $F_{i}(\mathrm{t})$ to choose when to exit. For the first price case, profits are

$$
\pi_{i}=v_{i} F_{1-i}(t)-c_{i}(t)
$$

Define $T_{i}$ by $c_{i}\left(T_{i}\right)=v_{i}$. Suppose, without loss of generality, that $T_{0} \geq T_{1}$. The unique solution to the first price war of attrition is:

$$
\text { For } 0 \leq t \leq T_{1}, \quad F_{0}(t)=c_{1}(t) / v_{1} ; \quad F_{1}(t)=\left[c_{0}(t)+v_{0}-c_{0}\left(T_{1}\right)\right] / v_{0}
$$

Firm 1, who has higher cost (at least at cost near value) has a mass point at 0 and earns zero profits. Firm 0 earns nonnegative expected profits, and the size of these profits are $v_{0}-c_{0}\left(T_{1}\right) .{ }^{9}$ Either player could be more likely to win the competition. It is demonstrated in the appendix that if firm 0 has uniformly lower marginal cost relative to value, ${ }^{10}$ it will win the competition with probability at least

$$
\frac{1}{2}\left[1+\left(1-\frac{c_{0}\left(T_{1}\right)}{v_{0}}\right)^{2}\right]
$$

The oral version of the war of attrition (see, e.g. Bulow and Klemperer, 1999) is also easy to describe using the same notation. Suppose firm $i$ is considering exiting at time $t$. The value of remaining in the game a bit longer is the value of winning times the probability that the opponent drops out, given that the opponent hasn't yet dropped so far. The cost of a slight delay in exiting is $c_{i}^{\prime}(t)$. Thus, a firm is indifferent to remaining in the game or exiting if

$$
\frac{v_{i} F_{1-i}^{\prime}(t)}{1-F_{1-i}(t)}-c_{i}^{\prime}(t)=0 \text {. }
$$

\footnotetext{
${ }^{9}$ If $v_{1}=0$, it may be necessary to "split zero," that is, introduce two values of zero, one less than the other, so that firm 0 can still choose $\mathrm{t}=0$ and win every time. Splitting zero is necessary for the solution to many games, and is most familiar in the solution to the Hotelling problem, where the two firms locate at the same point, one to the right of the other, and in the Bertrand pricing game with asymmetric costs, where one firm charges "just below" the other's cost. With a discrete action space, the necessity of splitting points goes away, but the model becomes cluttered with integer issues.

${ }^{10}$ Specifically, if $\mathrm{c}_{0}^{\prime}(\mathrm{t}) / \mathrm{v}_{0} \leq \mathrm{c}_{1}^{\prime}(\mathrm{t}) / \mathrm{v}_{1}$, then the claim holds. Generally, low cost and high value have the same effects.
} 
The pair of equations is solvable for a unique mixed strategy. In addition, it is well-known that there are other equilibria, because the decision to drop out is self-fulfilling. If player $i$ decides to drop out at $t_{i}$, then player $1-i$ rationally chooses to remain in the game, expecting player $i$ to exit in the next instant. ${ }^{11}$ However, the mixed strategy is unique. ${ }^{12}$ As a consequence, conditional on the war of attrition occurring, (2) holds with equality.

The oral version of the war of attrition has the following defect. Conditional on observing a war of attrition, the lower cost player is more likely to drop out. This defect arises for the usual reason with mixed strategy equilibria -- each player randomizes in such a way as to make the other player indifferent. As a consequence, the low cost player must be more likely to drop out in the next instant, so as to make it worth the cost to the high cost player of remaining in the game. It might be that appropriately chosen refinements insure the high cost player drops out more often at the start of the game. However, having the high cost player drop out initially doesn't solve the application problem. The theory still predicts that, when a war of attrition is observed, it is the low cost player who is more likely to exit, and the high cost player more likely to win the war. Fudenberg and Tirole (1986) note this defect, describing it as a consequence of mixed strategies, without additional comment.

From an economic perspective, the defect in the theory arises because the low cost player is forbidden by assumption from fully exploiting its low cost. The low cost player might like to present a show of force so large that the high cost player is forced to exit, but the usual game prohibits such endogenous effort. In most actual wars of attrition, players have the ability to increase their effort, so as to force the other side out. The US theory on war since Vietnam is that the public won't stand for a protracted conflict, and thus the US will lose if it does not win quickly. As a consequence, the US brings an overwhelming force to a conflict. (See, e.g. Correll, 1993.) The 1991 Desert Storm conflict appears to be an example of this approach. Similarly, Barnes and Noble entered internet book sales aggressively, with a large commitment of resources.

In the next section, a model of the war of attrition with endogenous effort is introduced. It works like a repeated version of the all-pay auction. Unlike the standard models, with endogenous effort, it is necessary to keep track of the state of the system over time, because the player exerting more effort is

\footnotetext{
${ }^{11}$ Strategies require saying what a player will do in any event; thus a player planning to exit at $t$ must still choose what it will do if it finds itself in the game at $t+1$. We produce multiplicity of equilibria by assuming that player $i$ will exit whenever it finds itself in the game after time $t_{i}$. This way, the opponent rationally believes that $i$ 's exit is coming immediately, even when the time exceeds $t_{i}$. Some of these equilibria require unreasonable beliefs and could likely be refined away.

${ }^{12}$ One player might exit at time 0 with strictly positive probability; conditional on not exiting at time 0 , the mixed strategy is unique.
} 
gaining an advantage over its rival that persists. The model is very similar to the tug of war model introduced by Harris and Vickers in 1987. There are three significant differences. Harris and Vickers use what Avinash Dixit (1987) calls a contest for the subgame, while an all-pay auction is used here. ${ }^{13}$ Second, discounting is permitted in the present analysis, and it turns out that discounting is very important, in that the limit of the solution, as players' common discount factors converge to unity, is degenerate. These differences in modeling permit the third major difference in the analysis: a closed form solution for the stationary equilibrium, and consequently greater insight into the comparative statics of the analysis, are available with the present model.

In their analysis, Harris and Vickers emphasize the combination of strategic interaction with uncertainty. Their stage game features uncertainty in the outcome for any given levels of effort by the players. In contrast, the present study has a deterministic outcome at the stage game; the player supplying greater effort wins. Uncertainty is endogenous: the deterministic stage game outcome induces randomization in the actions of the players. Depending on the application, either model might be more appropriate.

\section{The Continuing War of Attrition}

Two agents, named Left and Right, play a game over a set of states or nodes, indexed by $n=0,1, \ldots, N$. The game space is illustrated in Figure 1. When either extreme node 0 or $N$ is reached, the game ends. Payoffs for the players are $u_{0}$ and $v_{0}$, for Left and Right respectively, when node 0 is reached, and $u_{N}, v_{N}$ if node $N$ is reached. Reaching the right node $N$ is a win for Left, and a loss for Right. Conversely, reaching the node 0 is a win for Right. ${ }^{14}$ To formalize the notion of winning, assume:

$$
v_{0}>0>u_{0} \text { and } v_{N}<0<u_{N} .
$$

There will be discounting and a possibility that the game never ends, resulting in a zero payoff. Thus, (3) requires that winning is preferred to delay, and delay preferred to losing. While such an assumption was not required by the standard theories, which do not involve discounting, it seems reasonable that, faced with an inevitable loss, players would prefer to delay and hence discount the loss.

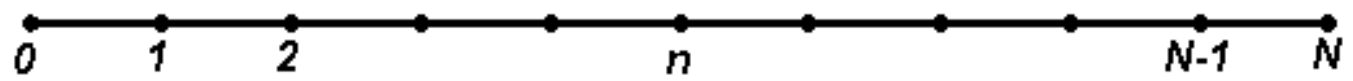

\section{Figure 1: The Playing Field}

\footnotetext{
${ }^{13}$ In addition to Dixit, Hershel Grossman (1990), Michelle Garfinkel (1991) and Sergio Skaperdas (1992) provide contest models to analyze some issues considered here.

${ }^{14}$ Notation will be such that Left's variables occur to the left of Right's variables in the alphabet.
} 
Consider a civil war in the nation of Chile, with the north fighting the south. Viewed from Argentina, the north is on the right hand side, while the south is on the left, and the country is reasonably viewed as a line segment. At any point in time, the battle point is a latitude line dividing the country. If the north wins a battle, it pushes the front southward (left), and wins the war when it wins battles all the way to Tierra Del Fuego.

At each node, the players play a first-price war of attrition. Strategies for the players are nonnegative effort levels. Denote by $x$ and $y$ the effort levels of Left and Right. The state transition is given by:

$$
n \rightarrow\left\{\begin{array}{cl}
n & \text { if } x=y \\
n+1 & \text { if } x>y \\
n-1 & \text { if } x<y
\end{array}\right.
$$

Thus, when Left exerts more effort, the node is advanced, and conversely when Right exerts more effort. The cost of $x$ and $y$ are set at $x$ and $y .{ }^{15}$ If the game ends at time T at node $n \in\{0, N\}$, Left's payoff will be of the form

$$
\boldsymbol{\delta}^{T} u_{n}-\sum_{t=0}^{T} \boldsymbol{\delta}^{t} x_{t}
$$

Right's payoff is analogous. In principle, the players' expected payoffs could depend on the entire history of the game, which can be arbitrarily long. However, with finitely many nodes, there will typically be a stationary equilibrium where each player has an expected value that depends only on the current state. Such stationary equilibria seem natural in this context and the analysis will focus on them. ${ }^{16}$

The analysis begins with the stage game. Suppose Left and Right use the distributional strategies $F_{n}$ and $G_{n}$ at node $n$. Denote by $u_{n}$ and $v_{n}$ the two player's continuation values at node $n$. Then, ignoring ties for the moment,

$$
u_{n}=\delta u_{n+1} G_{n}(x)+\delta u_{n-1}\left(1-G_{n}(x)\right)-x
$$

Similarly,

$$
v_{n}=\delta v_{n-1} F_{n}(y)+\delta v_{n+1}\left(1-F_{n}(y)\right)-y
$$

There are two kinds of equilibria to the stage game. First, there is a regular equilibrium, described in the previous section. In addition, there may be a degenerate equilibrium with both players choosing zero.

\footnotetext{
${ }^{15}$ If costs are linear, setting marginal costs at unity is without loss of generality, because $u_{0}$ and $u_{N}$ or $v_{0}$ and $v_{N}$ can be scaled to produce an equivalent optimization problem with unit marginal cost. The equilibrium analysis holds for convex costs, provided each cost function is a scalar multiple of the other, so that rescaling produces identical costs. It is potentially important that a tie leaves the state unchanged, rather than randomly selecting another state.

${ }^{16}$ There may be other equilibria. In particular, there are situations where both players prefer a draw with zero effort to continued war. Moreover, a draw could be supported with a threat of a return to hostilities (positive effort) in the event that a player defects. However, this turns out to be a stationary equilibrium without resorting to history dependence.
} 
Lemma 1: An equilibrium at node $n$ exists if and only if

$$
\begin{aligned}
& u_{n}-\delta u_{n-1} \geq 0 \\
& v_{n}-\delta v_{n+1} \geq 0 \\
& \text { At least one of (6), (7) holds with equality, } \\
& \bar{x}=\delta u_{n+1}-u_{n}=\delta v_{n-1}-v_{n} \geq 0
\end{aligned}
$$

In addition, $v_{n}=\delta v_{n+1}$ implies $v_{n} \leq 0$, and $u_{n}=\delta u_{n-1}$ implies $u_{n} \leq 0$. Finally, $u_{n}$ is strictly increasing in $n$ provided $u_{n} \neq 0$.

All proofs are contained in the Appendix.

Lemma 1 sets out the calculation of the stage game equilibrium, which was already presented in the previous section. Inequalities (6) and (7) describe the net payoffs from the stage game to the two players, and (8) requires that at least one of them must get zero net payoff. (The net payoff for Left is $u_{n}-\delta u_{n-1}$, which is the profit when $x=0$.) This is exactly analogous to the observation that at least one of the players in the one shot game is indifferent between playing the game and losing for sure. In the one-shot game, the net utility of one player is zero, and the other player's utility is computed using the requirement that the maximum of the support of the effort distribution is the same for both players. Equation (9) gives the maximum of the support, which is defined by the requirement that a certainty of a win doesn't change the net utility of the player. Given that (6) holds with equality, (9) gives the utility of Right.

The general form of equilibrium is illustrated in Figure 2. On the left, between $n=0$ and $L_{0}, v_{n}>0$. This implies (7) can't hold with equality, so that (6) does. As a consequence, $u_{n}=\delta u_{n-1}$. This equation is readily solvable by induction, and gives:

$$
u_{n}=\underline{u}_{n} \equiv \delta^{n} u_{0} \quad \text { for } n \leq L_{0} .
$$

Using (9), which is the equation that requires the upper endpoint of the support of the effort choices to be the same, we can also compute $v_{n}$, although with $n$ through $L_{0}-1$, because (9) uses $n+1$.

$$
v_{n}=\bar{v}_{n} \equiv \delta^{n}\left[v_{0}+n\left(1-\delta^{2}\right) u_{0}\right] \quad \text { for } n \leq L_{0}-1 .
$$

Similarly, to the right of $H_{0}$,

$$
\begin{aligned}
& v_{n}=\underline{v}_{n} \equiv \delta^{N-n} v_{N} \quad \text { for } n \geq H_{0}, \text { and, } \\
& u_{n}=\bar{u}_{n} \equiv \delta^{N-n}\left[u_{N}+(N-n)\left(1-\delta^{2}\right) v_{N}\right] \quad \text { for } n \geq H_{0}+1 .
\end{aligned}
$$


Equations (10)-(13) introduce some functions that are very important for the analysis of equilibrium behavior. The functions $\underline{u}_{n}, \underline{v}_{n}$ in equations (10) and (12), provide the minimum utility that the players can obtain. Equation (10) sets out the worst that can happen to Left. At node $n$, if Left invests nothing in the next $n$ battles, Left will lose the game $n$ periods hence, resulting in utility $\underline{u}_{n}$. Equation (12) is analogous.

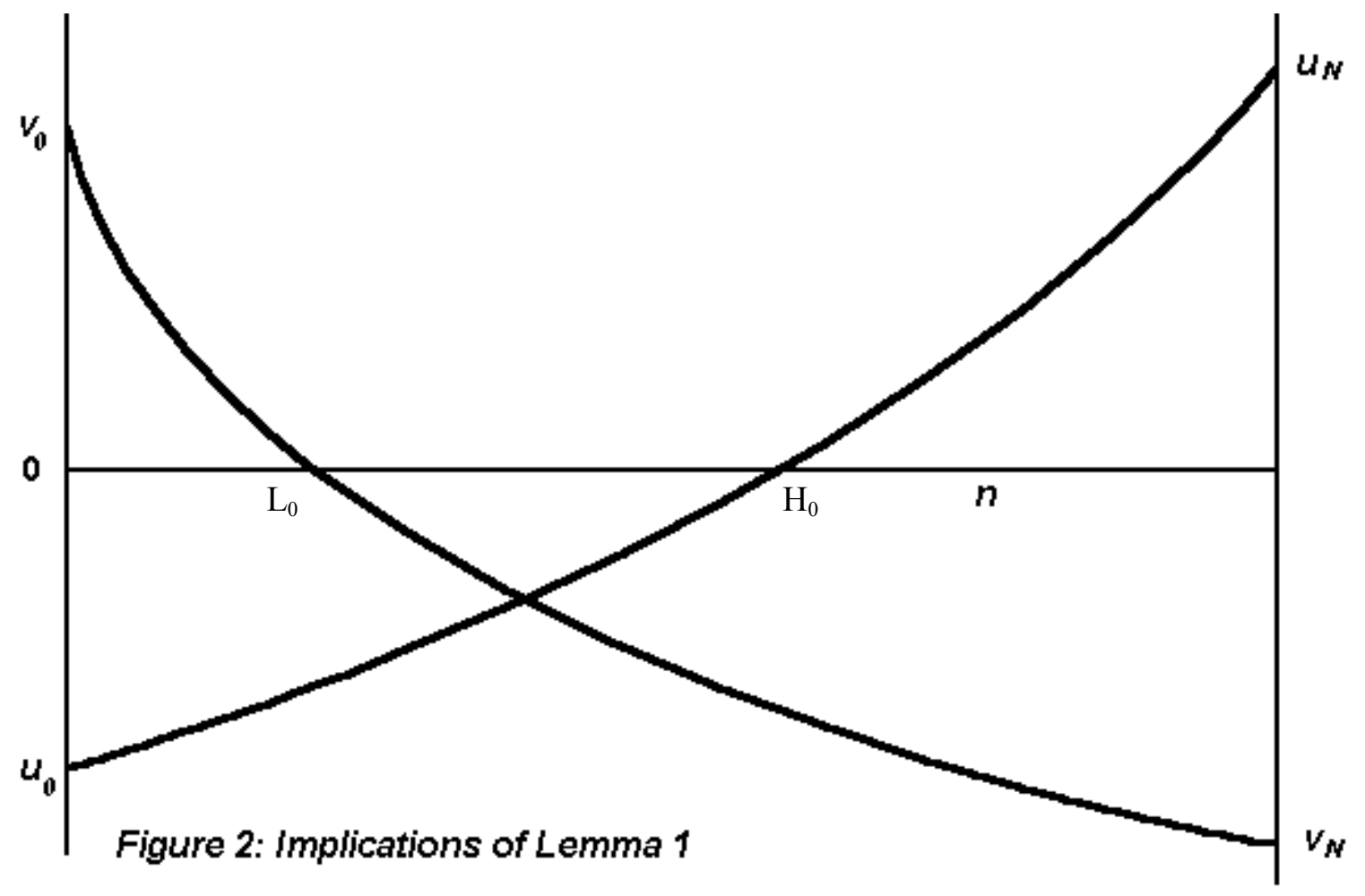

Given (10), it is possible to compute Right's payoff. This calculation is exactly analogous to the calculation of agent 0 's payoff in the static first-price war of attrition. Once we know that Left obtains zero net utility, we calculate Right's utility by examining the upper endpoint of the support, which is given in (9). We see in (11) that Right's payoff is composed of two terms. The first term is the utility of winning, which is discounted by the minimum number of periods it will take to reach the prize. This is not to say that Right will reach the prize in $n$ periods, but rather that it can, by exerting sufficient effort. The total effort exerted to win for sure, from position $n$, is $-\delta^{n} n\left(1-\delta^{2}\right) u_{0}$. In fact, the maximum effort at node $m$ is $-\delta^{m}\left(1-\delta^{2}\right)$, and discounting and summing gives the present value of the cost of effort of $-\delta^{n} n(1-$ $\left.\delta^{2}\right) u_{0}$. This outcome would arise if Right exerted maximum effort until winning. ${ }^{17}$

\footnotetext{
${ }^{17}$ It is not an equilibrium for Right to do so; Right must randomize. If it turned out, however, that the outcomes of the randomizations were there the maximum of the supports, then the outcome described arises, which gives Right's utility.
} 
Equations (10)-(13) do not necessarily fully characterize the equilibria. One could, in principle, imagine that which of (6) and (7) will bind with equality switches back and forth for $n$ between $L_{0}$ and $H_{0}$. However, it turns out that there are at most two switches, and if there are two of them, there is a (possibly degenerate) string of zero utilities in between. This gives a full characterization of the possible equilibria. To prove this, we need a preliminary lemma.

Lemma 2: Suppose $v_{n}=\delta v_{n+1}$ and $u_{n+1}=\delta u_{n}$. Then $u_{n} \leq v_{n}$ and $u_{n+1} \geq v_{n+1}$. In addition, if $v_{n-1}=\delta v_{n}, \mathrm{u}_{\mathrm{n}}=\mathrm{v}_{\mathrm{n}}$. If $u_{n+2}=\delta u_{n+1}, u_{n+1}=v_{n+1}$. In either case, $u_{n+1}=v_{n+1}=0$.

Figure 3 illustrates the situation where (6) holds with equality to the left of a point, $n^{*}$, and (7) holds to the right. Lemma 2 considers the reverse, where (7) holds with equality to the left, and (6) holds with equality to the right. The lemma shows that this can only happen once, at the point where $u_{n}=v_{n}$. In addition, if there is a sequence of these intermediate cases, it is associated with zero utility for both players. This case, with zero utility, is illustrated below in Figure 4.

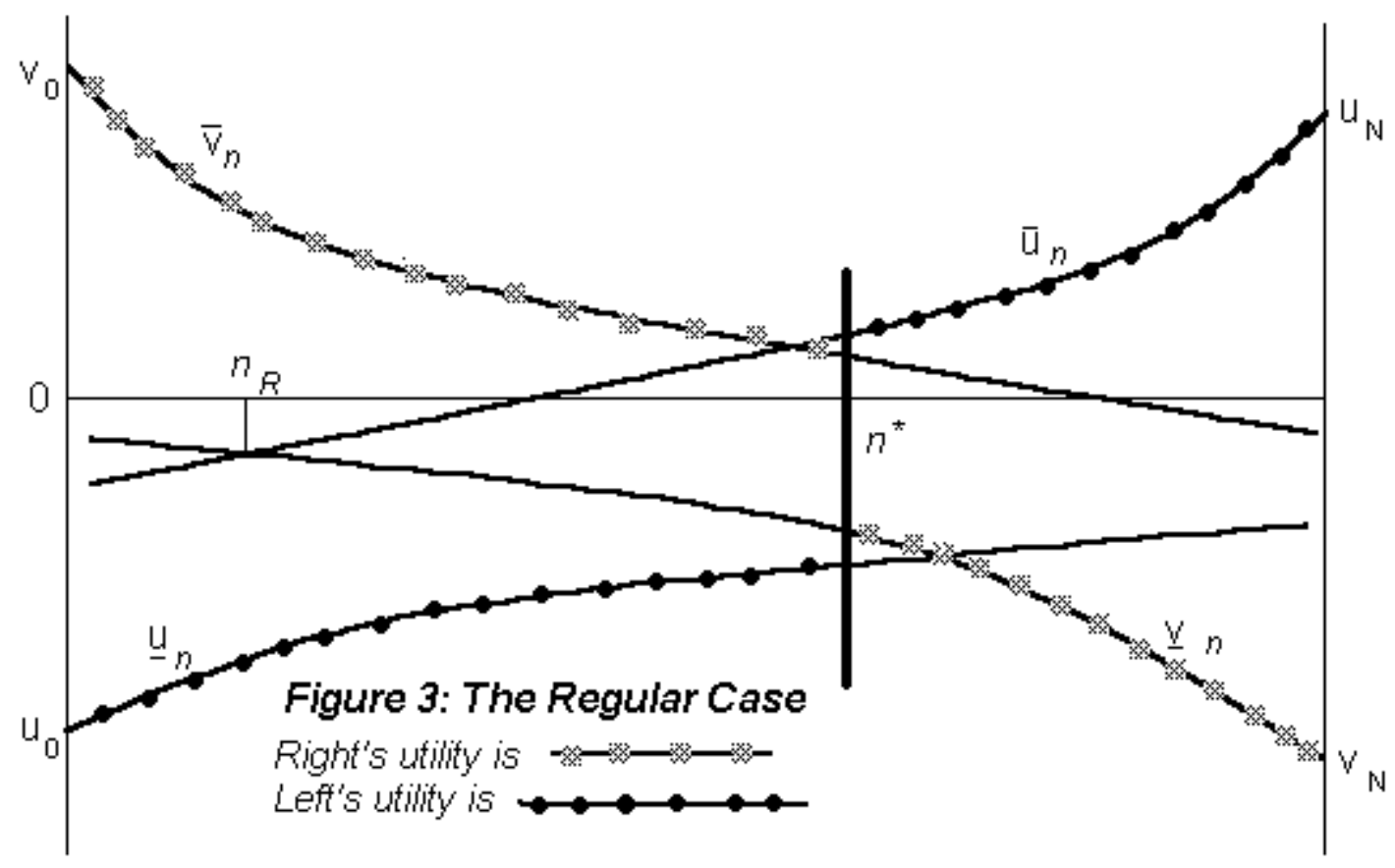

It may seem odd that $u_{n}=v_{n}$ defines the switching point in Lemma 2, since both player's utilities are potentially in different units. However, since the two player's marginal costs of effort have been set at unity by rescaling utility, and the maximum effort wins, there is a comparability between the utilities of the two players. Moreover, it turns out that $u_{n}=v_{n}$ defines a switching point--the sum of utilities is minimized when $u_{n}=v_{n}$ occurs. 
Figure 3 shows the general form of the regular case. In this case, all of the values of $u_{n}$ and $v_{n}$ are given by equations (10)-(13), with the exception of the two values on either side of the cross-over point $n$ *, which is the point where equations (10)-(11) give way to equations (12)-(13).

The difference between the regular case and the other case is that equations (10) and (11) intersect to the left of the point where equations (12) and (13) intersect. Such a situation makes Figure 3 impossible. In this case, there is a range of $n$ where neither player has strictly positive utility. This case is illustrated in Figure 4. In this case, the solution involves a sequence of points where the utility of both agents is zero. As the first theorem will show, this case arises when the discount factor is sufficiently low.

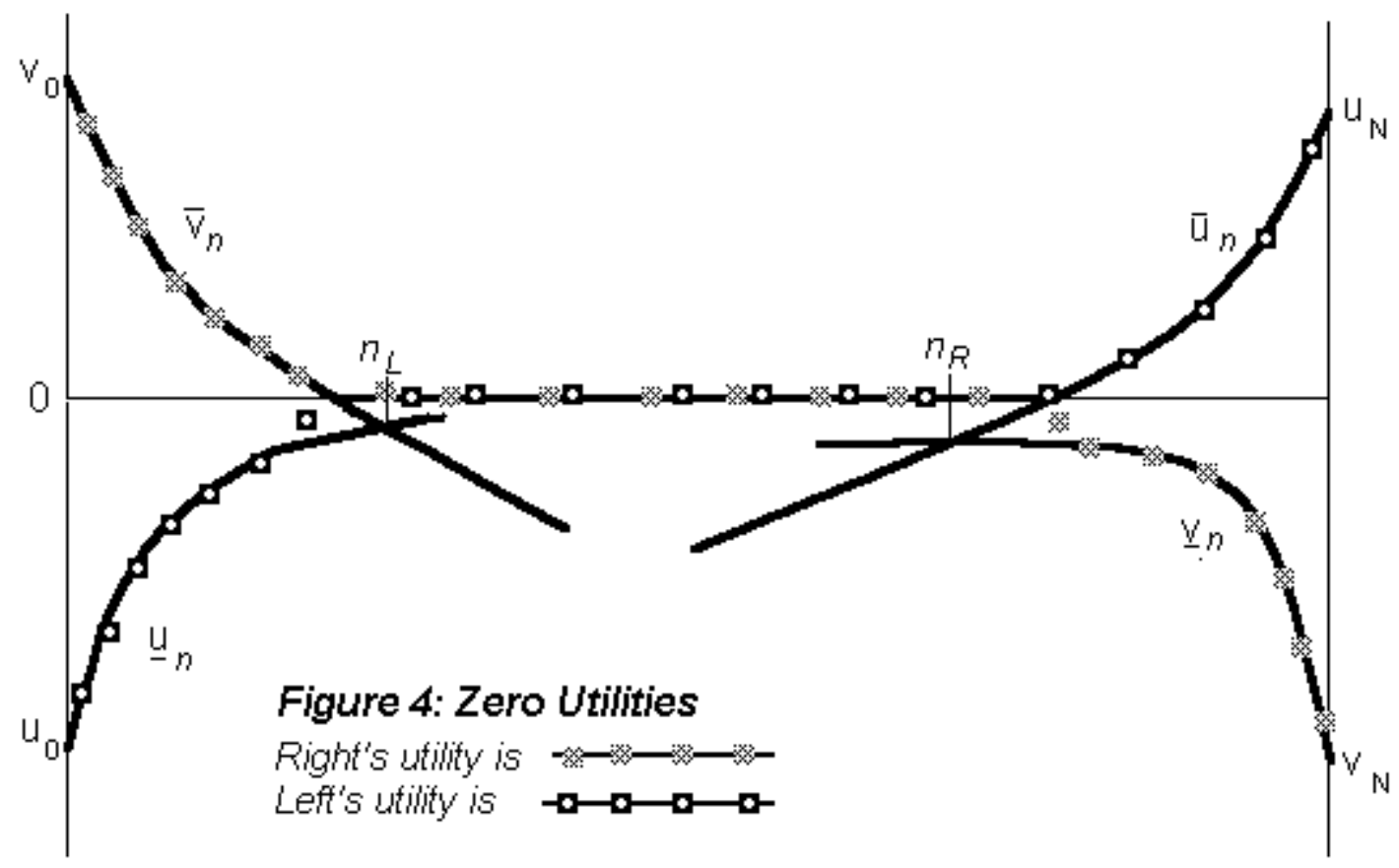

As a preliminary to the theorem, denote the intersections of the utility curves by $n_{L}$ and $n_{R}$, defined by

$$
\begin{aligned}
& \underline{u}_{n_{L}}=\bar{v}_{n_{L}} \\
& \underline{v}_{n_{R}}=\bar{u}_{n_{R}}
\end{aligned}
$$

Both Figures 3 and 4 have $n_{R}$ on them. It is not possible to display $n_{L}$ on Figure 3 because it occurs to the right of $N$. The two cases are distinguished by whether $n_{L}<n_{R}$ or not.

In addition, define

$$
\psi_{n}=\delta^{n}\left[u_{0}+v_{0}+n\left(1-\delta^{2}\right) u_{0}\right]
$$




$$
\phi_{n}=\delta^{N-n}\left[u_{N}+v_{N}+(N-n)\left(1-\delta^{2}\right) v_{N}\right]
$$

The functions $\psi_{n}$ and $\phi_{n}$ give the sum of the agents' utilities in the case when $n$ is sufficiently low ( $\left.n \leq L\right)$ and $n$ sufficiently high $(n \geq H)$. The function $\psi_{n}$ has the property that it decreases in $n$ until $n_{L}$, at which point $\psi_{n}$ is negative, and then increases. ${ }^{18}$ The function $\phi_{n}$ is analogous.

It is useful to define a real number $n^{*}$ by $\psi_{n^{*}}=\phi_{n^{*}}$; with $n^{*}=0$ if $\psi_{0} \leq \phi_{0}$, and $n^{*}=N$ if $\psi_{N} \leq \phi_{N}$. If $n_{L} \leq n_{R}$ or $n^{*}$ is not in $\left[n_{R}, n_{L}\right]$, then $n^{*}$ is said not to exist.

\section{Theorem 1: (Existence and characterization of equilibrium)}

(a): If $n^{*}$ exists and $\psi_{n^{*}} \geq 0$, then there is a unique stationary equilibrium, with

$$
v_{n}=\left\{\begin{array}{ll}
\bar{v}_{n} & \text { if } n \leq L-1 \\
\underline{v}_{n} & \text { if } n \geq L+1
\end{array} \quad u_{n}= \begin{cases}\underline{u}_{n} & \text { if } n \leq L \\
\bar{u}_{n} & \text { if } n \geq L+2\end{cases}\right.
$$

and $\psi_{L-1} \geq \phi_{L+1}$ and $\psi_{L} \leq \phi_{L+2}$. /L-n*/ $\leq 1$.

(b): If $n^{*}$ exists and $\psi_{n^{*}}<0$, there are two stationary equilibria, one as in part (a), one with a sequence of zeros as in part (c) below.

(c): If $n *$ does not exist, then there is a unique stationary equilibrium, defined by $\psi_{L-1} \geq 0>\psi_{L}$ and $\phi_{H}<0 \leq \phi_{H+1}$, with $L<H$, and

$$
v_{n}=\left\{\begin{array}{ll}
\bar{v}_{n} & \text { if } n \leq L-1 \\
0 & \text { if } L+1 \leq n \leq H-1 \\
\underline{v}_{n} & \text { if } n \geq H
\end{array} \quad u_{n}= \begin{cases}\underline{u}_{n} & \text { if } n \leq L \\
0 & \text { if } L+1 \leq n \leq H-1 \\
\bar{u}_{n} & \text { if } n \geq H+1\end{cases}\right.
$$

The three cases considered in Theorem 1 are not exhaustive; however, they cover most of the specifications of parameters. In addition, the Theorem does not provide the exact calculations where the utilities are not given by (10)-(13). These calculations are provided in the Appendix at some considerable tedium.

\footnotetext{
${ }^{18}$ Specificially, $\psi_{n} \geq \psi_{n+2}$ if and only if $\bar{v}_{n+2} \geq \underline{u}_{n+2}$. This occurs if and only if $n+2 \leq n_{L}$. There are integer issues throughout the analysis, such as this one. Because such issues vanish in the limit as the number of points grows, they will be ignored in the text, with exact calculations provided in the Appendix.
} 
Theorem 1 shows that there are three major cases. First, if the payoffs are such that $\psi_{n}$ and $\phi_{n+2}$ intersect at a positive value, then the equilibrium is unique and looks like the description provided in Figure 3, with one agent receiving positive utility up to a switch point $n^{*}$, then the other agent receiving positive utility. The second type of equilibrium involves a string of zeros in the central portion of the graph. This type of equilibrium is unique, if $\psi_{n}=\phi_{n}$ either doesn't solve or solves outside the region where $\psi_{n}$ is decreasing and $\phi_{n}$ is increasing. For an intermediate range of parameters, both types of equilibria exist.

Let $p_{n}$ denote the probability of the transition $n \rightarrow n-1$, which is a win for Right. The following lemma computes $p_{n}$.

Lemma 3: For $0<n<N$,

$$
p_{n}=\left\{\begin{array}{ll}
1-1 / 2 \frac{-u_{0}}{v_{0}+(n-1) u_{0}-\delta^{2}(n+1) u_{0}} & \text { if } n<L \\
1 / 2 \frac{-v_{N}}{u_{N}+(N-n-1) v_{N}-\delta^{2}(N-n+1) v_{N}} & \text { if } n>H
\end{array} .\right.
$$

In all equilibria, a portion of the equilibrium behavior is described by equations (10)-(13). A number of interesting observations follow directly from these equations, and Lemma 2.

Theorem 2: (a): In any equilibrium, the sum of utilities is u-shaped, initially declining in $n$ and then rising (possibly with a segment constant at zero), and

(b): Average and maximum efforts are $u$-shaped,

(c): The probability that Left wins a battle is less than $1 / 2$ for $n<L$, and greater than $1 / 2$ for $n>H$.

(d): The probability that Left wins a battle is increasing in $n$, for $n<L$ and $n>H$.

Theorem 2 has the following implications. There is a defense disadvantage--the agent closest to losing the war is more likely to lose any given battle. In particular, when the battlefront is near Left's home base, Left wins the next battle with probability less than 50\%. In spite of this likelihood of losing, the closer the current node gets to the end, the harder both sides fight. Utility is maximized on the edges, and minimized in the center. Finally, there is a momentum effect. As Left gets closer to winning, it's likelihood of winning the next battle rises. This effect is a consequence of discounting, and doesn't arise if the players do not discount future payoffs. 
Remark 1: There can be situations where neither side fights. These must arise when the discount factor is low. If so, the sum of utilities is non-negative.

Remark 2: There can be equilibria where, for some nodes, both players have negative expected value. Such equilibria exhibit a prisoner's dilemma feature. If dropping out of the game is permitted, then the players would like to do this, but not if dropping out means losing the war.

How does the probability of winning a battle translate into the probability of winning the war? Let $q_{n}$ denote the probability that Left wins the war, that is, the state reaches $N$. The probabilities $q_{n}$ are defined by $q_{0}=0, q_{N}=1$, and

$$
q_{n}=p_{n} q_{n-1}+\left(1-p_{n}\right) q_{n+1} \text {. }
$$

Equation (19) expresses the law of motion for translating the likelihoods of winning battles into ultimate victory in the war. For economy of expression, it is configured so that $p_{n}$ is the probability Left loses a battle, but $q_{n}$ is the probability Left wins the war. Equation (19) states that the likelihood of winning from state $n$ is the likelihood of winning from state $n-1$, times the probability of reaching that state, plus the likelihood of winning from state $n+1$ weighted by the probability of transition to that state.

A second object of interest is the duration of the war. Let $\Delta_{n}$ denote the expected duration of the war. Analogous to $q_{n}$, the expected duration satisfies $\Delta_{0}=\Delta_{N}=0$ and

$$
\Delta_{n}=1+p_{n} \Delta_{n-1}+\left(1-p_{n}\right) \Delta_{n+1} .
$$

Lemma 4: The probability that Left wins, $q_{n}$, and the expected conflict duration, $\Delta_{n}$, satisfy:

$$
\begin{aligned}
q_{n}=\frac{\sum_{k=0}^{n-1}\left(\prod_{j=1}^{k} \frac{p_{j}}{1-p_{j}}\right)}{\sum_{k=0}^{N-1}\left(\prod_{j=1}^{k} \frac{p_{j}}{1-p_{j}}\right)} . \\
\Delta_{n}=q_{n} \sum_{m=0}^{N-1} \sum_{j=1}^{m} \frac{1}{p_{j}}\left(\prod_{k=j}^{m} \frac{p_{k}}{1-p_{k}}\right)-\sum_{m=0}^{n-1} \sum_{j=1}^{m} \frac{1}{p_{j}}\left(\prod_{k=j}^{m} \frac{p_{k}}{1-p_{k}}\right)
\end{aligned}
$$

Formulas (21) and (22) give exact solutions to the likelihood that Left wins the war of attrition, and the expected length of time that war will persist.

Theorem 3: The probability that Left wins the war, $q_{n}$, is nondecreasing in $n$. In addition, $q_{n}$ is concave for $n<L$ and convex for $n>H$. 
While it is comforting to have exact calculations such as those in (21) and (22), the discrete model is cumbersome. Not only are the formulas complex, but there are integer issues that will vanish in the limit. The rest of this section considers the limiting model played on a continuous interval.

In the limiting case, we fix

$$
e^{-\beta}=\delta^{N}
$$

and then send $N$ to infinity. Equation (23) fixes the amount of discounting required to cross the entire playing field, so that the set of points is refined, in effect holding the overall distance constant. It is unnecessary to reduce the costs of conflict, since that is equivalent to scaling utilities. Let $\lambda=n / N$. Then the analogues of equations (16) and (17) are

$$
\psi(\lambda)=e^{-\beta \lambda}\left(u_{0}+v_{0}+2 \beta u_{0} \lambda\right),
$$

and,

$$
\phi(\lambda)=e^{-\beta(1-\lambda)}\left(u_{N}+v_{N}+2 \beta v_{N}(1-\lambda)\right) .
$$

The analogues to $n_{L}$ and $n_{R}$ are the values of $\lambda$ that minimize $\psi$ and $\phi$, respectively. The intersection of these curves, at $\lambda^{*}$, can occur at either a positive or negative value of $\psi$. The value $\lambda^{*}$ is the limit of $n^{*}, L$ and $H$, when these are close. The analogue to Theorem 1 is:

Remark 3: For the limit as $N \rightarrow \infty$, with (23) holding, there are at most two limits of stationary equilibria. If $\lambda *$ exists, there is an equilibrium with the sum of utilities equal to $\max \{\psi(\lambda), \phi(\lambda)\}$. If $\psi\left(\lambda^{*}\right)<0$ or if $\lambda^{*}$ does not exist, there is an equilibrium with the sum of utilities equal to $\max \{\psi(\lambda), \phi(\lambda), 0\}$; this equilibrium is unique if $\lambda^{*}$ does not exist.

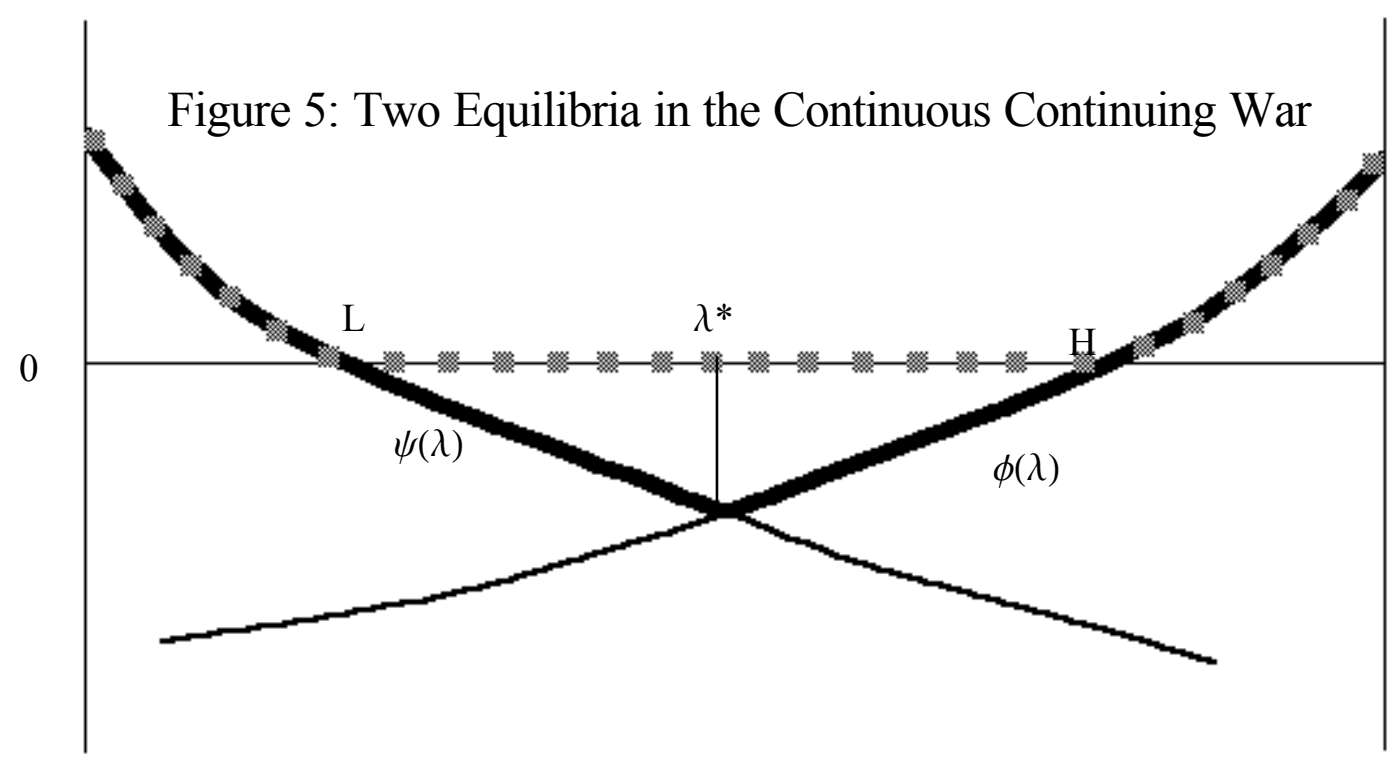


Figure 5 illustrates the sum of utilities in the case where there are two equilibria. One equilibrium involves negative sum of utility in the central portion and follows the thick, dark line. This equilibrium does not end in a draw, and is the victor is determined by whether the game starts to the left of $\lambda^{*}$ or not. In the other equilibrium, there is a draw between $L$ and $H$. Effort at any given node of course converges to zero as the number of nodes diverges. However, the flow rate of effort does not vanish other than in a draw.

Theorem 4: In the limit of regular equilibria, $n / N \rightarrow \lambda, N \rightarrow \infty$,

$$
p_{n} \rightarrow p(\lambda)= \begin{cases}1-1 / 2 \frac{-u_{0}}{v_{0}-2 u_{0}+2 \lambda \beta u_{0}} & \text { if } \lambda<\lambda * \\ 1 / 2 \frac{-v_{N}}{u_{N}-2 v_{N}+2 \lambda \beta v_{N}} & \text { if } \lambda>\lambda *\end{cases}
$$

$$
q_{n} \rightarrow q(\lambda)= \begin{cases}0 & \text { if } \lambda<\lambda * \\ 1 & \text { if } \lambda>\lambda *\end{cases}
$$

$$
\frac{\Delta_{n}}{N} \rightarrow \begin{cases}\int_{0}^{\lambda} \frac{1}{2 p(x)-1} d x & \text { if } \lambda<\lambda * \\ \int_{\lambda}^{1} \frac{1}{1-2 p(x)} d x & \text { if } \lambda>\lambda *\end{cases}
$$

With a very fine grid, the likelihood of winning any particular battle for Left converges to a number which is not zero or one, but in between. However, since the war is now composed of a very large number of battles, the outcome of the war is deterministic. The duration of the war converges to a remarkably simple expression, and is roughly linear in $n / N{ }^{19}$ The duration can be interpreted as follows. With a likelihood of moving left of $p_{n}$, the expected net leftward movement per period is $p_{n}-\left(1-p_{n}\right)=2 p_{n}-1$. Thus, $1 /\left(2 p_{n}-1\right)$ is the expected number of periods to move one unit to the left. The integral to the current period gives the number of periods to reach zero. The analogous calculation holds to the right of $\lambda^{*}$.

While the continuing war of attrition is more complicated than the standard models, due to the endogenous effort, the continuing version does have relatively simple limiting behavior. Moreover, we

\footnotetext{
${ }^{19}$ To be precise, duration is exactly linear when there is no discounting $--\beta=0$. In this case, $p$ is constant (although a different constant on either side of $\left.\lambda^{*}\right)$.
} 
can perform a number of thought experiments that were not available in the standard versions of the model.

\section{Comparative Statics and Special Cases}

\section{Lower Cost of Effort}

A lower cost of effort is equivalent to rescaling both the utility of winning and losing by a factor exceeding unity. For example, if Left's cost of effort is reduced in half, the effect is the same as doubling $u_{0}$ and $u_{N}$. Lowering the cost of Left's effort shifts $\psi$ down and $\phi$ up, and thus shifts $n^{*}$ to the left unambiguously. The region where Left is more likely to win expands. The effect on $n^{*}$ is illustrated in Figure 6 for the continuous case. In addition, the likelihood that Left wins any particular battle also increases, as we see from (18). In the limit, the duration of the war falls when Left is the likely winner, and rises when Left is the likely loser.

One of the main undesirable features of the standard models of the war of attrition is the prediction that the low cost player is more likely to lose. In contrast, the continuing war of attrition does have the low cost player more likely to win, and even more likely the lower is the player's cost. This feature is very important for any application.

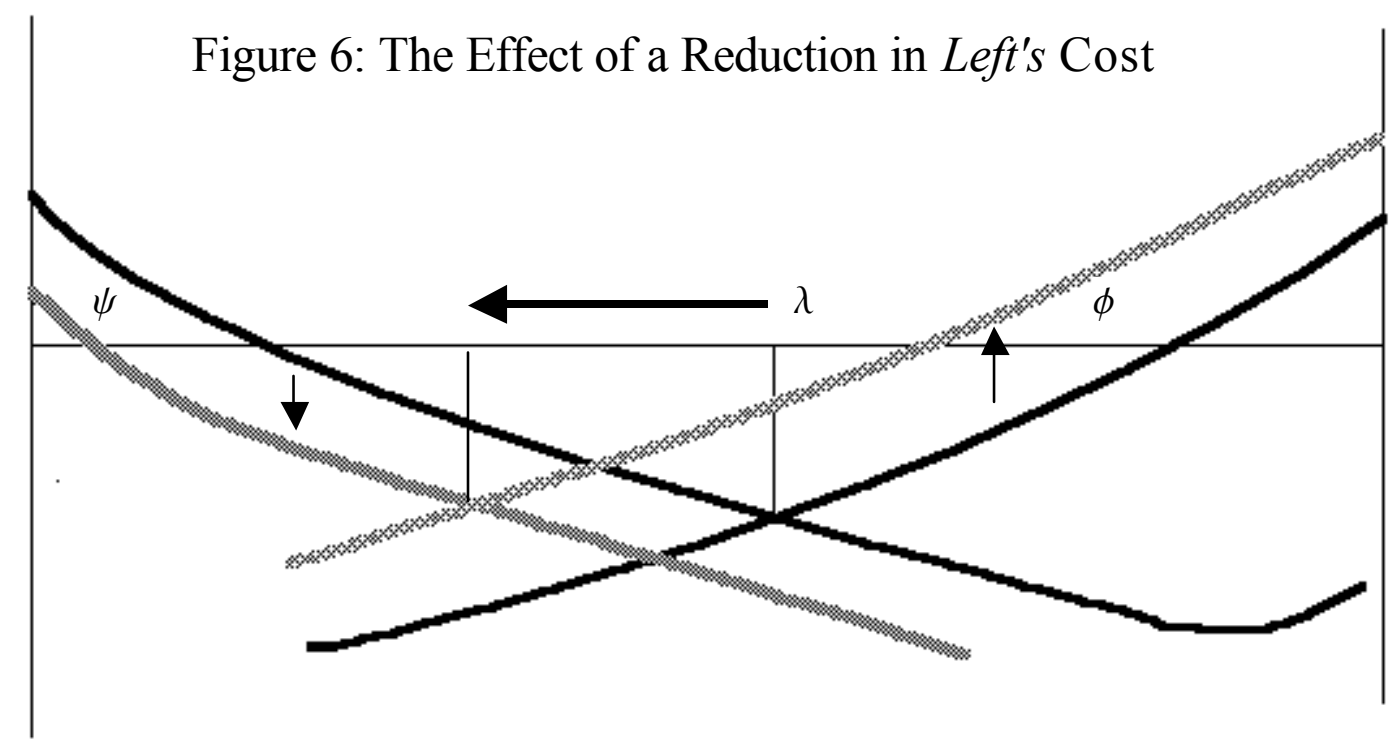

A reduction in Left's cost uniformly increases Left's chance of winning the conflict, starting from any point. If there is a region in which the two don't fight in equilibrium, that region may grow or shrink, but shifts leftward. The expected duration of the conflict rises in the area where Right is likely to win (to the left of $\left.\lambda^{*}\right)$, and is reduced in the area that Left is likely to win. 


\section{The Colonial Power}

Contests such as the US in Vietnam, France in Algeria or Microsoft versus Netscape may be considered as analogous to a colonial war; one side continues to survive after a loss in the conflict, while the other is extinguished. We let the larger power be Right, with the defenders being Left. A victory for Right implies that Left is extinguished; the cost to Left of a loss should be viewed as being very large. This willingness to suffer any consequence to avoid losing might be modelled as $u_{0}$ going to $-\infty$. As $u_{0} \rightarrow-\infty$, so does $\psi$, and thus the region where Right wins disappears. Here, unless the colonial power wins an instant victory, it loses.

However, the desire of the defenders not to lose is not the only salient aspect of a colonial war. The colonial power typically has a lower cost of materials, and perhaps even of troops, given a larger population. Lowering the cost of fighting is the same as a rescaling of the values of winning and losing. Thus, sending the cost of fighting to zero sends both $v_{0}$ and $-v_{N}$ to $\infty$. As we saw above, this favors the colonial power, and the prediction of the likely winner turns on whether the cost of the colonial power is relatively low, when compared with the cost of the defenders.

\section{Patient Players}

When players can take actions very often, the time between battles is reduced and the discount factor converges to 1 . This is a different thought experiment than making the playing field continuous while holding the discounting required to cross the entire playing field constant. It is easy to see that $\psi_{n} \rightarrow u_{0}+v_{0}$ and $\phi_{n} \rightarrow u_{N}+v_{N}$, so that there is a global winner, and it is the agent whose victory provides the greatest combined surplus. If that combined surplus is negative for either winner, then there will also be an equilibrium where there is a draw, except on the edges.

If the original game (ignoring effort) is constant sum, so that $u_{0}+v_{0}=u_{N}+v_{N}$, so that the combined surplus is the same for both players, then the switch point $n^{*}$ satisfies:

$$
\frac{n^{*}}{N}=\frac{u_{N}-v_{N}}{u_{N}-v_{N}+v_{0}-u_{0}} .
$$

Thus, the relative profits from victory determine the critical point, and Left is more likely to win the larger is his profits. 


\section{Legal Battles}

Consider a tort dispute between a potentially injured plaintiff and a potentially liable defendant. ${ }^{20}$ We let the plaintiff be Left and the defendant be Right. An important characteristic of the legal system is that a win for the defendant involves the same payoff as a draw without fighting; that is, the defendant pays the plaintiff nothing. Formally, in the model, $u_{0}=v_{0}=0$, implying $\psi_{n}=0$. The prediction of the theory is that there are two regions, with a draw on the left, and fighting on the right. Thus, rather than plaintiffs formally losing, the plaintiffs just go away when the situation favors the defendant.

If $u_{N}+v_{N}<0$, then a draw is the unique outcome. At first glance, it might seem that a legal dispute has to be a negative sum game. However, these values are scaled by the cost of effort, so that, when the plaintiff has a lower cost of effort, $u_{N}$ may well exceed $-v_{N}$, even when the original game is zero sum.

In order to win, a plaintiff needs to survive a number of challenges by the defendant. First, defendants regularly challenge the plaintiff's standing to sue. If the plaintiff survives, the defendant requests summary judgment -- that the plaintiff can't, as a matter of law, win. If the plaintiff wins that battle, the plaintiff is permitted to put on a case. At this point, the defendant typically requests a directed verdict, alleging that the plaintiff has failed to prove their case. Again, should the plaintiff prevail, the defendant puts on their case; if the plaintiff prevails, typically the defendant appeals. One can think of this as a five node battle (this means $\mathrm{N}=6$ ).

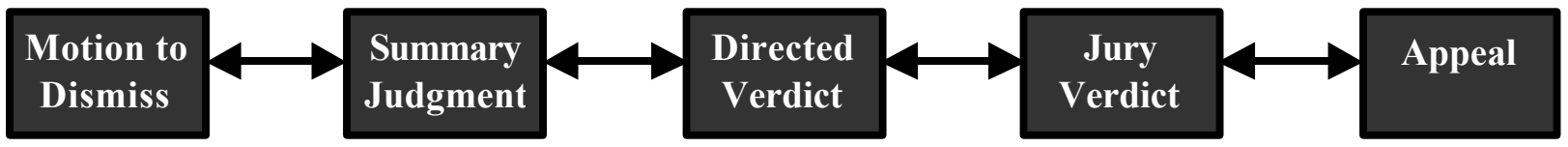

If the plaintiff loses at a stage, the plaintiff can appeal; victory in the appeal permits advancement to the right. $^{21}$ As a practical matter, if the plaintiff loses the jury verdict, appeal is relatively difficult. In the model, the current node is a sufficient statistic for the state of the system. In an actual legal conflict, as in many conflicts, history will matter -- even when an appeal sets aside a jury verdict, some of the issues (such as discovery limits) may remain.

\footnotetext{
${ }^{20}$ Litigation is often viewed as a war of attrition. For a particularly entertaining example, see David Kim's battles with Sotheby's auction house, at Supreme Court of New York (1997).

${ }^{21}$ In the event of a victory, the plaintiff can sell the rights to the judgment to the Judgment Purchase Corporation, who will then handle the appeal. See Fisk, 1999.
} 


\section{Conclusion}

The present model accounts for interesting features of the war of attrition. First, a lower cost of effort is an advantage. Second, there is what might be described as a momentum effect--as a player gets closer to winning, the player's likelihood of winning each battle, and the war, increases. Third, as a player gets closer to winning, and the other gets closer to losing, their efforts rise. Fourth, even in a model in which an infinitesimal effort can upset a tie, a draw is possible. Fifth, reducing a player's cost of effort will raise (lower) the expected conflict duration when that player is weaker (stronger) than his opponent.

That a lower cost of effort leads to a greater likelihood of victory seems like a necessary condition for a war of attrition to be a plausible model of asymmetric contests. While weakness can be an advantage in some conflicts, it is generally only advantageous when weakness induces accommodation, as in the puppy dog strategy of Fudenberg and Tirole, 1984. As the term war of attrition is commonly understood, accommodation is not an option.

As one player gets close to winning, it seems quite reasonable that efforts rise. The winning player has an incentive to try to end the war quickly, since that is feasible. Similarly, for the losing player, increased effort delays defeat and the consequent utility loss. That effort is maximized near the endpoints of the game provides for "last ditch efforts" on the part of the (losing) defender, and an attempt to finish the job on the part of the (winning) offense. However, the result that total effort is u-shaped is at least a little surprising. In particular, since there is a discontinuity in the payoff around $\lambda^{*}$, one might have expected a good bit more effort devoted to pivoting around this point, rather than a passive acceptance of being on the losing side of $\lambda^{*}$ on the part of the defense.

The existence of a draw is quite plausible, and appears to arise in actual conflicts, such as the Hundred Years War between France and Britain, which displayed long periods of a cessation of hostilities. In the Cold War between the United States and the Soviet Union, there was also a period of "peaceful coexistence," which could be interpreted as a draw. Theoretically, a draw should appear as a stationary equilibrium whenever it is too costly for one side to win when the other side devotes small levels of effort. Over the past 900 years, the position of Switzerland, relative to militarily strong neighbors, appears to fit this description. Switzerland had little value of winning a war against a neighbor, since it would be unlikely to succeed in extracting much surplus from a neighboring country. The militarily strong neighbor faced a difficult task to defeat Switzerland, because of the terrain (which creates a high 
cost of effort for an invading force), and, in this century, Switzerland's well-armed populace. As a consequence, the model appears to account for Swiss independence.

How can nations increase the likelihood of peaceful coexistence? The theory suggests that reducing the payoff to victory unambiguously increase the set of stable interior outcomes, which have a peaceful coexistence nature. Similarly, increasing the loss from defeat increases the set of peaceful coexistence nodes. These conclusions are reminiscent of the deterrence theory of warfare, which holds that deterrence arises when the balance of interests favors the defender. ${ }^{22}$ In particular, it is the relative value of the defender and attacker that determines the outcome. The logic is that if the defender values the territory more than the attacker, the defender will have a stronger will to persist; in this event the attacker will lose. Attackers backward induct and decide not to attack in such circumstances. This is precisely the prediction of the model, in that peaceful co-existence occurs at all nodes left of $\lambda^{*}$ when $u_{0}+v_{N}<0$, that is, the cost to the defender of losing exceeds the value to the attacker of winning. ${ }^{23}$

Rational deterrence theory has been severely criticized on the grounds that conflicts occur when the theory suggests that the conflicts are not in either party's interests. ${ }^{24}$ The present study suggests that multiple equilibria, one with deterrence or peaceful co-existence, one with war to the end, are a natural outcome in territorial disputes. The theory also suggests a distinction between strong deterrence, when peaceful co-existence is the unique equilibrium, and weak deterrence, when peaceful co-existence is one of two equilibria. Such a distinction may be useful in understanding the failures of rational deterrence theory. ${ }^{25}$

A reduction of the cost of effort for one side has an ambiguous effect on peaceful co-existence. In the model, a reduction in the cost of effort for both by equal amounts, should reduce the scope for peaceful co-existence. This also seems plausible. In some sense, the gain from conflict has not changed, but its cost has been reduced, so the likelihood of conflict ought to increase. The model, therefore, can capture the idea that new weapons can be destabilizing even when held by both sides of the conflict. Weapons

\footnotetext{
${ }^{22}$ See Achen and Snidal, 1989, for an eloquent discussion of the theory and its relation to real situations. Schelling, 1962, discusses the need for randomness in the outcome. As commonly employed, the theory requires each country to be represented by a rational representative agent, and these agents playing a full information game.

${ }^{23}$ Lieberman, 1995, uses the deterrence theory to account for the conflict between Egypt and Israel which followed shortly after the 6 day war, a conflict commonly called the War of Attrition (March, 1969-August 1970).

24 . See Achen and Snidal, 1989, for a summary and critique.

${ }^{25}$ However, it is not sensible to insist on a full-information rational agent theory. Deterrence theory has much in common with limit pricing theory, and the approach taken by Milgrom and Roberts, 1982, offers significant insights for rational deterrence theory, including an understanding of bluffing (via pooling equilibria), and signalling (sabre-rattling). Some of the critics of rational deterrence theory are actually criticizing an implicit full information assumption. Given the secrecy employed by the military, the full information assumption is inappropriate.
} 
such as the neutron bomb are sometimes considered to be defensive only and not offensive. While the model does not readily incorporate the distinction between defensive and offensive weapons, the effects may be modeled by presuming that defensive weapons increase the cost of effort. Such an change increases the set of stable nodes in the model.

When the value of winning is zero, all interior nodes are stable in the unique equilibrium. This no-win situation is the theory of mutually assured destruction for the model; by eliminating the value of winning, neither side has an incentive to fight, even when a player would like her opponent to lose to insure she doesn't lose.

The cost of effort has an ambiguous effect on the expected conflict duration. By making a strong (winning) player stronger, the player wins more a larger proportion of battles and the war ends more quickly, while the reverse is true when the weak player is made stronger, unless the weak player is made sufficiently stronger to become the strong player.

How well does the model confront the colonial conflicts, such as Microsoft versus Netscape, Barnes and Noble versus Amazon.com, the US in Vietnam, or the Union versus the Confederacy? In contrast to the standard model, being strong is an advantage. Moreover, a kind of momentum arises endogenously. Around the critical point $n^{*}$, small gains can make significant differences in the likelihood of winning the conflict. Indeed, in the limiting continuum solution, the likelihood of winning is discontinuous at $\lambda^{*}$. In the case of Internet Explorer or Amazon.com, network externalities are sometimes identified as a reason that there will be an eventual winner. In the present model, the extreme form of network externalities (winner-take-all) imposed as a primitive translates into a critical point at which there is a discontinuity in payoffs.

In the model, as one side gets near to winning, both sides fight harder. In the military environment, the mortality rate for soldiers should rise near the end of the conflict. This seems implausible for many conflicts. In a business context, this prediction should be testable; advertising should be u-shaped in market share, and prices should be as well. As Internet Explorer's market share rose, the prices of both browsers fell, eventually to zero. The Department of Justice lawsuit against Microsoft probably confounds later observations about effort by the parties, for Microsoft was given a reason to accommodate Netscape's Explorer. 
In colonial wars and market share fights, typically the holder of territory or market share derives a flow return roughly proportional to the market share. As a consequence, one might expect the contender in the lead to be able to devote more resources to the conflict, favoring that side. Such considerations appear to reinforce the instability of sharing the market. 


\section{References}

Achen, Christopher H. and Duncan Snidel, "Rational Deterrence Theory and Comparative Case Studies," World Politics 41, no. 2, January 1989, 143-69.

Amann, Erwin, and Wolfgang Leininger, "Asymmetric All-Pay Auctions with Incomplete Information," Games and Economic Behavior, 14, no. 1, May 1996, 1-18.

Baye, Michael, Daniel Kovenock and Caspar de Vries, "The All-Pay Auction with Complete Information," Economic Theory 8, Spring 1996, 291-305.

Bulow, Jeremy and Paul Klemperer, "The Generalized War of Attrition," American Economic Review, forthcoming.

Cannings, C. and J.C. Whittaker, "The Finite Horizon War of Attrition," Games and Economic Behavior, 11, no. 2, November, 1995, 193-236.

Correll, John T., " Tinkering With Deadly Force," Air Force Magazine, Vol.76, No. 1, January 1993. Can be found at http://www.afa.org/magazine/editorial/01edit93.html

Dixit, Avinash, "Strategic Behavior in Contests," American Economic Review, 77, no. 5, December, 1987, 891-8.

Fisk, Margaret, "Large Verdicts for Sale," National Law Journal, January 11, 1999.

Fudenberg, Drew, and Jean Tirole, "A Theory of Exit in Duopoly," Econometrica 54, no. 4, July, 1986, 943-960.

Fudenberg, Drew, and Jean Tirole, "The Fat Cat Effect, the Puppy Dog Ploy and the Lean and Hungry Look," American Economic Review, 74 (May 1984), 361-366.

Garfinkel, Michelle, "Arming as a Strategic Investment in a Cooperative Equilibrium," American Economic Review, 80, March 1990, 50-68.

Grossman, Herschel, "A General Equilibrium Model of Insurrections," American Economic Review, 81, September 1991, 912-21.

Harris, Christopher and John Vickers, "Racing with Uncertainty," Review of Economic Studies, LIV, 1987, 1-21.

Hendricks, Ken, and Charles Wilson, "The War of Attrition in Discrete Time," unpublished, August, 1995.

Hendricks, Ken, and Charles Wilson, "Equilibrium in Preemption Games with Complete Information," Equilibrium and Dynamics, ed: Mahul Majumdar, MacMillan Press, 1992, 123-47.

Hendricks, Ken, Andrew Weiss and Charles Wilson, "The War of Attrition in Continuous Time with Complete Information," International Economic Review, 29, no.4, November, 1988, 663-680.

Lieberman, Elli, "Deterrence Theory: Success or Failure in the Arab-Israeli Wars?," McNair Paper 45, National Defense University, Washington, D.C., October, 1995. 
Kapur, Sandeep, "Markov Perfect Equilibria in an N-Player War of Attrition," Economics Letters, 47, no.2, February, 1995, 149-54.

Kaserman, David L., and John W. Mayo, Government and Business: The Economics of Antitrust and Regulation," Orlando: Dryden Press, 1995, p.595-6.

Kautt, William, "The Anglo-Irish War--A People's War, 1916-1921," Westport, CT: Praeger Publishers, 1999. (Book currently unavailable; description at http://info.greenwood.com/books.)

Krishna, Vijay and John Morgan, "An Analysis of the War of Attrition and the All Pay Auction," Journal of Economic Theory, 72, no.2, February 1997, 343-62.

Mack, Andrew, "Why Big Nations Lose Small Wars," World Politics, 27, no. 2, January 1975, 175-200.

Milgrom, Paul, and John Roberts, "Limit Pricing and Entry under Incomplete Information: An Equilibrium Analysis," Econometrica 50, No. 2, March, 1982, pp. 443-460.

Milgrom, Paul and Robert Weber, "A Theory of Auctions and Competitive Bidding," Econometrica, 50, September 1982, 1089-1122.

Paret, Peter, "French Revolutionary Warfare from Indochina to Algeria: The Analysis of a Political and Military Doctrine," New York: Frederick A. Praeger, 1964.

Schelling, Thomas C., "Nuclear Strategy in Europe," World Politics 14, No. 3, April 1962, pp. 421-432.

Shirer, William The Rise and Fall of the Third Reich, New York: Simon and Schuster, 1959.

Skaperdas, Sergio, "Cooperation, Conflict and Power in the Absence of Property Rights," American Economic Review, 82, September, 1992, 720-39.

Smith, Maynard, "The Theory of Games and the Evolution of Animal Conflicts," Journal of Theoretical Biology, 47, 1974, 209-21.

Supreme Court of the State of New York, affidavit of David Yoon Kim, Feburuary 20, 1997, Index No. 107652/96. See description at http://www.sothebyssellskimfake.com/

Zebrowski, Carl, " Why the South Lost the Civil War," American History, 33, no. 6. Feb. 1999. (Also at http://www.thehistorynet.com/AmericanHistory/) 


\section{Appendix: Proofs and Derivations}

In the first price war of attrition, if firm 0 has uniformly lower marginal cost, it wins more often.

The probability that firm 0 wins is

$$
\begin{gathered}
\int_{0}^{T_{1}} F_{1}(t) F_{0}^{\prime}(t) d t=\left(v_{0}-c_{0}\left(T_{1}\right)\right) / v_{0}+\frac{1}{v_{0} v_{1}} \int_{0}^{T_{1}} c_{0}(t) c_{1}^{\prime}(t) d t \geq\left(v_{0}-c_{0}\left(T_{1}\right)\right) / v_{0}+\frac{1}{v_{0}^{2}} \int_{0}^{T_{1}} c_{0}(t) c_{0}^{\prime}(t) d t \\
=\left(v_{0}-c_{0}\left(T_{1}\right)\right) / v_{0}+\frac{c_{0}\left(T_{1}\right)^{2}}{2 v_{0}^{2}}=\frac{1}{2}\left(1+\left(1-\frac{c_{0}\left(T_{1}\right)}{v_{0}}\right)^{2}\right)
\end{gathered}
$$

\section{Proof of Lemma 1:}

Generally, equilibria will require mixing. There is a significant pure strategy equilibrium when

$$
v_{n-1}=v_{n}=u_{n}=u_{n+1}=0, v_{n+1} \leq 0 \text {, and } u_{n-1} \leq 0 .
$$

Both players play zero.

This is the only pure strategy possible. This can be observed from (4) and (5) and the observation that pure strategies must involve ties.

Now consider mixed strategies.

Note that the closure of the supports of the distributions of effort must coincide, for otherwise there are values chosen with higher cost and no higher likelihood of advancing the node. A similar argument shows that the closure of the support must be an interval. Moreover, if there is a mass point, it is at zero. To see this, note that if Left used a mass point at $x_{1}>0$, it would not be profitable for Right to choose $y$ such that $x_{1}-\epsilon<y<x_{1}$, since increasing $y$ to slightly more than $x_{1}$ provides a discrete jump in the likelihood of a favorable outcome with a continuous cost increase. But this means that Left can reduce $x_{1}$ by $\epsilon$ without any reduction in probability of success. This logic permits us to write

$$
F_{n}(x)=\frac{v_{n}-\delta v_{n+1}}{\delta\left(v_{n-1}-v_{n+1}\right)}+\frac{x}{\delta\left(v_{n-1}-v_{n+1}\right)}
$$

$$
G_{n}(y)=\frac{u_{n}-\delta u_{n+1}}{\delta\left(u_{n+1}-u_{n+1}\right)}+\frac{y}{\delta\left(u_{n+1}-u_{n-1}\right)}
$$

From the fact that both players can't have a mass point at zero (for otherwise it pays to resolve the ties in one's favor by an arbitrarily small additional expenditure), we obtain (6), (7) and (8). Solving for the upper bound of the support gives (9).

Suppose $u_{n}=\delta u_{n-1}$. Then the maximum of the support at node $n-1$ satisfies

$0 \leq \delta u_{n}-u_{n-1}=\left(\delta^{2}-1\right) u_{n-1}$. 
Finally, $u_{n-1}<0$ implies $u_{n} \geq \delta u_{n-1}>u_{n-1} . u_{n-1}>0$ implies $u_{n-1} \leq \delta u_{n}<u_{n}$. Thus, $u_{n}$ is increasing in $n$, except perhaps when $u_{n}=0$. (Either inequality shows $u_{n}$ is weakly increasing when $u_{n}=0$.

Q.E.D.

Derivation of Formulas (10)-(13):

Eq. (10) follows immediately from (7) holding with equality, which arises because $v_{n}>0$. From (9), $v_{n}=\delta v_{n-1}+u_{n}-\delta u_{n+1}=\delta v_{n-1}+\delta^{\mathrm{n}}\left(1-\delta^{2}\right) u_{0}$,

which readily gives (11). (12) and (13) are symmetric. Q.E.D.

\section{Proof of Lemma 2:}

From (9), $u_{n}=v_{n}+\delta u_{n+1}-\delta v_{n-1}=\delta^{2} u_{n}+v_{n}-\delta v_{n-1} \leq \delta^{2} u_{n}+v_{n}-\delta^{2} v_{n}$, which reduces to $u_{n} \leq v_{n}$, with equality when $v_{n-1}=\delta v_{n}$.

Similarly, at node $n+1,(9)$ gives

$u_{n+1}-v_{n+1}=\delta u_{n+2}-\delta v_{n}=\delta u_{n+2}-\delta^{2} v_{n+1} \geq \delta^{2}\left(u_{n+1}-v_{n+1}\right)$, with equality if $u_{n+2}=\delta u_{n+1}$. Q.E.D.

\section{Proof of Theorem 1:}

From Lemma 2, there are three types of candidates for a stationary equilibrium. The proof proceeds by analyzing each case individually, and then showing that the theorem holds.

Case 1: $u_{n}=\delta u_{n-1}$ for $n \leq L$ and $v_{n}=\delta v_{n+1}$ for $n \geq L+1$

Case 2: $u_{n}=\delta u_{n-1}$ for $n \leq L, v_{L+1}=\delta v_{L+2}, u_{L+2}=\delta u_{L+1}$, and $v_{n}=\delta v_{n+1}$ for $n \geq L+3$

Case 3: $u_{n}=\delta u_{n-1}$ for $n \leq L, v_{L+1}=\delta v_{L+2}, u_{n}=0$ for $L+1 \leq n<H, u_{H+1}=\delta u_{H}$, and $v_{n}=\delta v_{n+1}$ for $n \geq H+2$

In case 1, there is no constraint reversal as hypothesized by Lemma 2, so that (10)-(13) hold with $n_{H}=n_{L}+1$. In case 2 , there is a constraint reversal as hypothesized by Lemma 2 , but no run of such reversals. Lemma 2 demonstrates that a run of reversals yields zero utility for both players; that case is considered in case 3 .

Case 1: It will prove useful to define two functions of $n$

(A4) $\psi_{n}=\delta^{n}\left[u_{0}+v_{0}+n\left(1-\delta^{2}\right) u_{0}\right]$

(A5) $\phi_{n}=\delta^{N-n}\left[u_{N}+v_{N}+(N-n)\left(1-\delta^{2}\right) v_{N}\right]$

Note that $\psi_{n}$ gives the sum of utilities for $n \leq L-1$, while $\phi_{n}$ gives the sum of utilities for $n \geq L+1$.

Equations (10) - (13) give values of $u_{n}$ and $v_{n}$ for all but two instances: $u_{L+1}$ and $v_{L}$. Equation (9), for $n=L$, $L+1$ gives

$$
\left(\begin{array}{cc}
1 & \delta \\
\delta & 1
\end{array}\right)\left(\begin{array}{c}
v_{L} \\
u_{L+1}
\end{array}\right)=\left(\begin{array}{c}
\underline{u}_{L}+\delta \bar{v}_{L-1} \\
\underline{v}_{L+1}+\delta \bar{u}_{L+2}
\end{array}\right)=\delta\left(\begin{array}{l}
\psi_{L-1} \\
\phi_{L+2}
\end{array}\right)
$$

which gives 


$$
\left(\begin{array}{c}
v_{L} \\
u_{L+1}
\end{array}\right)=\frac{\delta}{1-\delta^{2}}\left(\begin{array}{c}
\psi_{L-1}-\delta \phi_{L+2} \\
\phi_{L+2}-\delta \psi_{L-1}
\end{array}\right)
$$

There are three types of constraints, all from Lemma 1. By construction, both (8) and the equality in (9) hold. The remaining constraints are:
1a) $\quad u_{n}-\delta u_{n-1} \geq 0$ for $n \geq L+1$
1b) $\quad v_{n}-\delta v_{n+1} \geq 0$ for $n \leq L$
1c) $\quad \delta u_{n+1}-u_{n} \geq 0$, for $1 \leq n \leq N-1$.

There are six conditions that are equivalent to case 1 comprising an equilibrium.

$$
\begin{array}{ll}
\text { 1.1: } & \left(1-\delta^{2}\right) \phi_{L+3}+\delta^{2} \psi_{L-1}-\phi_{L+1} \geq 0 . \\
\text { 1.2: } & \left(1-\delta^{2}\right) \psi_{L-2}+\delta^{2} \phi_{L+2}-\psi_{L} \geq 0 . \\
\text { 1.3: } & \phi_{L+2} \geq \psi_{L} \\
\text { 1.4: } & \phi_{L+1} \leq \psi_{L-1} \\
\text { 1.5: } & \phi_{L+4} \geq \phi_{L+2} \\
\text { 1.6: } & \psi_{L-1} \leq \psi_{L-3}
\end{array}
$$

Case 1, part a). 1.1, 1.3, and 1.5 are necessary and sufficient for part a).

For $n \geq L+3$,

$$
\begin{aligned}
u_{n}-\delta u_{n-1}= & \delta^{N-n}\left[u_{N}+(N-n)\left(1-\delta^{2}\right) v_{N}\right]-\delta^{N-n+2}\left[u_{N}+(N-n+1)\left(1-\delta^{2}\right) v_{N}\right] \\
& =\delta^{N-n}\left(1-\delta^{2}\right)\left[u_{N}+(N-n) v_{N}-\delta^{2}(N-n+1) v_{N}\right] \\
& =\delta^{N-n}\left(1-\delta^{2}\right)\left[u_{N}-v_{N}+(N-n+1)\left(1-\delta^{2}\right) v_{N}\right] \\
& =\frac{1-\delta^{2}}{\delta}\left(\bar{u}_{n-1}-\underline{v}_{n-1}\right) \\
& =\delta\left(\phi_{n+1}-\phi_{n-1}\right)
\end{aligned}
$$

which is nonnegative for $n \geq L+3$ if and only if 1.5.

For $n=L+2, \quad u_{L+2} \geq \delta u_{L+1}$

if and only if

if and only if

$$
\left(1-\delta^{2}\right) \bar{u}_{L+2}-\delta^{2}\left(\phi_{L+2}-\delta \psi_{L-1}\right) \geq 0
$$

if and only if

$$
\left(1-\delta^{2}\right) \bar{u}_{L+2}-\delta^{2}\left(\phi_{L+2}-\delta \phi_{L+1}\right)-\delta^{2}\left(\delta \phi_{L+1}-\delta \psi_{L-1}\right) \geq 0
$$

if and only if

$$
\left(1-\delta^{2}\right) \bar{u}_{L+2}-\left(1-\delta^{2}\right) \delta \bar{u}_{L+1}-\delta^{3}\left(\phi_{L+1}-\psi_{L-1}\right) \geq 0
$$

if and only if

$$
\left(1-\delta^{2}\right)\left[\bar{u}_{L+2}-\delta \bar{u}_{L+1}\right]-\delta^{3}\left(\phi_{L+1}-\psi_{L-1}\right) \geq 0
$$




$$
\left(1-\delta^{2}\right) \phi_{L+3}+\delta^{2} \psi_{L-1}-\phi_{L+1} \geq 0
$$

This is 1.1

If $n=L+1, u_{L+1} \geq \delta u_{L}$

if and only if $\quad \delta\left(\phi_{L+2}-\delta \psi_{L-1}\right) \geq \delta\left(1-\delta^{2}\right) \underline{u}_{L}$

if and only if $\quad \phi_{L+2} \geq \delta \psi_{L-1}+\left(1-\delta^{2}\right) \underline{u}_{L}=\psi_{L}$.

This condition both follows from, and implies, 1.3.

Part 1b) This part is symmetric with part 1a.

Part 1c) For $n \leq L-1, \delta u_{n+1}-u_{n}=\delta^{n} u_{0}\left(\delta^{2}-1\right)>0$.

For $n=L$,

$$
\begin{aligned}
\delta u_{L+1}-u_{L} & =\frac{\delta^{2}}{1-\delta^{2}}\left(\phi_{L+2}-\delta \psi_{L-1}\right)-\delta^{L} u_{0} \\
& =\frac{\delta^{2}}{1-\delta^{2}} \phi_{L+2}-\frac{\delta^{2}}{1-\delta^{2}}\left(\psi_{L}-\delta^{L}\left(1-\delta^{2}\right) u_{0}\right)-\delta^{L} u_{0} \\
& =\frac{\delta^{2}}{1-\delta^{2}}\left(\phi_{L+2}-\psi_{L}\right)+\delta^{L}\left(\delta^{2}-1\right) u_{0}>0
\end{aligned}
$$

For $n=L+1$,

$$
\begin{aligned}
\delta u_{L+2}-u_{L+1} & =\delta^{N-L-1}\left(u_{N}+(N-L-2)\left(1-\delta^{2}\right) v_{N}\right)-\frac{\delta}{1-\delta^{2}}\left(\phi_{L+2}-\delta \psi_{L-1}\right) \\
& =\frac{\delta^{N-L-1}}{1-\delta^{2}}\left[\left(u_{N}+(N-L-2)\left(1-\delta^{2}\right) v_{N}\right)\left(1-\delta^{2}\right)-\left(u_{N}+v_{N}+(N-L-2)\left(1-\delta^{2}\right) v_{N}\right)\right]+\frac{\delta^{2}}{1-\delta^{2}} \psi_{L-1} \\
& =-\frac{\delta^{N-L-1}}{1-\delta^{2}}\left[\delta^{2}\left(u_{N}+(N-L-2)\left(1-\delta^{2}\right) v_{N}\right)+v_{N}\right]+\frac{\delta^{2}}{1-\delta^{2}} \psi_{L-1} \\
& =-\frac{\delta^{N-L-1}}{1-\delta^{2}}\left[\delta^{2}\left(u_{N}+v_{N}+(N-L-1)\left(1-\delta^{2}\right) v_{N}\right)+\left(1-\delta^{2}\right)^{2} v_{N}\right]+\frac{\delta^{2}}{1-\delta^{2}} \psi_{L-1} \\
& =\frac{\delta^{2}}{1-\delta^{2}}\left(\psi_{L-1}-\phi_{L+1}\right)-\delta^{N-L-1}\left(1-\delta^{2}\right) v_{N}>0 .
\end{aligned}
$$

For $n \geq L+2$,

$$
\begin{aligned}
\delta u_{n+1}-u_{n} & =\delta \delta^{N-n-1}\left[u_{N}+(N-n-1)\left(1-\delta^{2}\right) v_{N}\right]-\delta^{N-n}\left[u_{N}+(N-n)\left(1-\delta^{2}\right) v_{N}\right] \\
& =-\left(1-\delta^{2}\right) \delta^{N-n} v_{N}>0
\end{aligned}
$$

Finally, the above analysis must be verified for $L \epsilon\{0, N\}$. It is a routine computation to show there is an equilibrium with $L=0$ whenever $\phi_{N-2}-\psi_{0} \geq 0$. Similarly, $L=N$ arises when $\psi_{N-2}-\phi_{0} \geq 0$. These are the natural generalizations of the interior cases. 
Case 2: Let $s_{n}=u_{n}+v_{n}$. Note that $u_{n}=\delta u_{n-1}$ if and only if $s_{n-1} \geq s_{n+1}$. It follows from the description of case 2 , that

$$
\begin{aligned}
& s_{L-1} \geq S_{L+1}, \\
& s_{L} \leq s_{L+2}, \\
& s_{L+1} \geq s_{L+3}, \\
& s_{L+2} \leq s_{L+4} .
\end{aligned}
$$

Thus, in particular,

$$
\begin{aligned}
& \psi_{L-1}=s_{L-1} \geq s_{L+1} \geq s_{L+3}, \text { and } \\
& s_{L} \leq s_{L+2} \leq s_{L+4}=\phi_{L+4} .
\end{aligned}
$$

We will use these to show that $\psi_{L-1} \geq \phi_{L+3}$ and $\psi_{L} \leq \phi_{L+4}$, so that there is a crossing of $\psi$ and $\phi$ around $L$, just as in case 1 .

The proof of case 2 begins with the calculation of the agents' utilities. From Lemma 1, these satisfy:

$$
\begin{aligned}
& v_{L}+\delta u_{L+1}=u_{L}+\delta v_{L-1}=\delta \psi_{L-1}, \\
& v_{L+1}-\delta v_{L+2}=0 \\
& \delta u_{L+1}-u_{L+2}=0 \\
& \delta v_{L}-v_{L+1}+u_{L+1}-\delta u_{L+2}=0 \\
& \delta v_{L+1}-v_{L+2}+u_{L+2}-\delta u_{L+3}=0 \\
& \delta v_{L+2}+u_{L+3}=v_{L+3}+\delta u_{L+4}=\delta \phi_{L+4} .
\end{aligned}
$$

In matrix form, these equations yield

$$
\left(\begin{array}{cccccc|c}
1 & 0 & 0 & \delta & 0 & 0 \\
0 & 1 & -\delta & 0 & 0 & 0 & v_{L} \\
0 & 0 & 0 & \delta & -1 & 0 & v_{L+1} \\
\delta & -1 & 0 & 1 & -\delta & 0 & v_{L+2} \\
0 & \delta & -1 & 0 & 1 & -\delta \\
0 & 0 & \delta & 0 & 0 & 1
\end{array}\right)=\left(\begin{array}{c}
\delta \psi_{L+1} \\
u_{L+2} \\
u_{L+3}
\end{array}\right)\left(\begin{array}{c}
0 \\
0 \\
0 \\
0 \\
\phi_{L+4}
\end{array}\right)
$$

Thanks to Mathematica, rewrite this as

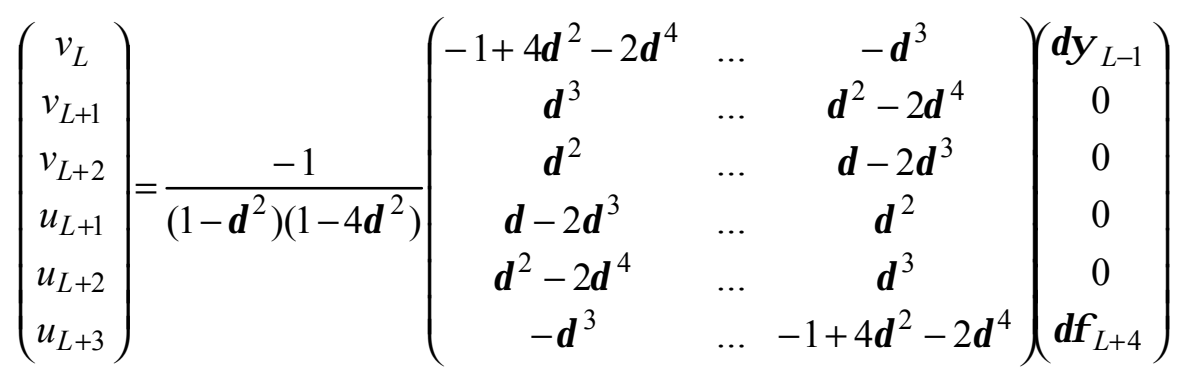


Thus,

$$
=\frac{-\delta}{\left(1-\delta^{2}\right)\left(1-4 \delta^{2}\right)}\left(\begin{array}{c}
-\left(1-4 \delta^{2}+2 \delta^{4}\right) \psi_{L-1}-\delta^{3} \phi_{L+4} \\
\delta^{3} \psi_{L-1}+\delta^{2}\left(1-2 \delta^{2}\right) \phi_{L+4} \\
\delta^{2} \psi_{L-1}+\delta\left(1-2 \delta^{2}\right) \phi_{L+4} \\
\delta\left(1-2 \delta^{2}\right) \psi_{L-1}+\delta^{2} \phi_{L+4} \\
\delta^{2}\left(1-2 \delta^{2}\right) \psi_{L-1}+\delta^{3} \phi_{L+4} \\
-\delta^{3} \psi_{L-1}-\left(1-4 \delta^{2}+2 \delta^{4}\right) \phi_{L+4}
\end{array}\right)
$$

$$
\begin{aligned}
s_{L}= & u_{L}+v_{L} \\
= & \delta^{L} u_{0}-\frac{\delta}{\left(1-\delta^{2}\right)\left(1-4 \delta^{2}\right)}\left(-\left(1-4 \delta^{2}+2 \delta^{4}\right) \psi_{L-1}-\delta^{3} \phi_{L+4}\right) \\
& \frac{\delta}{\left(1-\delta^{2}\right)\left(1-4 \delta^{2}\right)}\left(\left(1-\delta^{2}\right)\left(1-4 \delta^{2}\right) \delta^{L-1} u_{0}+\left(1-4 \delta^{2}+2 \delta^{4}\right) \psi_{L-1}+\delta^{3} \phi_{L+4}\right) \\
& =\frac{\delta}{\left(1-\delta^{2}\right)\left(1-4 \delta^{2}\right)}\left(\frac{1-4 \delta^{2}}{\delta} \psi_{L}+2 \delta^{4} \psi_{L-1}+\delta^{3} \phi_{L+4}\right) \\
& =\frac{\psi_{L}}{1-\delta^{2}}+\frac{\delta^{4}}{\left(1-\delta^{2}\right)\left(1-4 \delta^{2}\right)}\left(2 \delta \psi_{L-1}+\phi_{L+4}\right) .
\end{aligned}
$$

Similarly,

and

$$
\begin{aligned}
& s_{L+1}=u_{L+1}+v_{L+1}=\frac{-\delta}{\left(1-4 \delta^{2}\right)}\left(\delta \psi_{L-1}+2 \delta^{2} \phi_{L+4}\right), \\
& s_{L+2}=u_{L+2}+v_{L+}=\frac{-\delta}{\left(1-4 \delta^{2}\right)}\left(2 \delta^{2} \psi_{L-1}+\delta \phi_{L+4}\right),
\end{aligned}
$$

$$
\begin{aligned}
s_{L+3} & =u_{L+3}+v_{L+3} \\
& =\delta^{N-L-3} v_{N}+\frac{\delta}{\left(1-\delta^{2}\right)\left(1-4 \delta^{2}\right)}\left(\delta^{3} \psi_{L-1}+\left(1-4 \delta^{2}+2 \delta^{4}\right) \phi_{L+4}\right) \\
& =\frac{\phi_{L+3}}{1-\delta^{2}}+\frac{\delta^{4}}{\left(1-\delta^{2}\right)\left(1-4 \delta^{2}\right)}\left(\psi_{L-1}+2 \delta \phi_{L+4}\right) \\
& =\frac{\phi_{L+3}}{1-\delta^{2}}-\frac{\delta^{2} s_{L+1}}{1-\delta^{2}}
\end{aligned}
$$

Thus, $s_{L+1} \geq s_{L+3}$ if and only if $s_{L+1} \geq \phi_{L+3}$. Combined with (A6), this yields $\psi_{L-1} \geq \phi_{L+3}$. A similar argument generates $s_{L} \leq s_{L+2}$ if and only if $\psi_{L} \leq s_{L+2}$, which combines with (A7) to give $\psi_{L} \leq \phi_{L+4}$.

Finally, from Lemma $2, u_{L+1} \leq v_{L+1}$ and $u_{L+2} \geq v_{L+2}$. Thus

$$
u_{L+1} \leq v_{L+1}=\delta v_{L+2} \leq \delta u_{L+2}=\delta^{2} u_{L+1},
$$

and thus $u_{L+1}$ and $v_{L+1}$ are negative. Thus $0 \geq s_{L+1} \geq \psi_{L+1}$. A similar argument shows that $0 \geq s_{L+2} \geq \phi_{L+2}$. Thus, the intersection of $\psi_{n}$ with $\phi_{n+2}$ occurs with $\psi_{n}<0$.

Case 3: For $\mathrm{L}>1$, in case 3, we know from Lemma 2 that $v_{L+1}=\delta v_{L+2}=0=u_{L+2}$. The equality in (9) gives 
$u_{L}-v_{L}=\delta u_{L+1}-\delta v_{L-1}$ and $u_{L+1}-v_{L+1}=\delta u_{L+2}-\delta v_{L}$

Thus $u_{L+1}=-\delta v_{L}$.

$\delta \psi_{L-1}=u_{L}+\delta v_{L-1}=\delta u_{L+1}+v_{L}=\left(1-\delta^{2}\right) v_{L}$.

3a)

$$
\begin{aligned}
u_{L+1}-\delta u_{L} & =-\frac{\delta^{2}}{1-\delta^{2}} \delta^{L-1}\left[u_{0}+v_{0}+(L-1)\left(1-\delta^{2}\right) u_{0}\right]-\delta^{L+1} u_{0} \\
& =-\frac{\delta^{L+1}}{1-\delta^{2}}\left[u_{0}+v_{0}+(L-1)\left(1-\delta^{2}\right) u_{0}\right]-\delta^{L+1} u_{0} \\
& =-\frac{\delta}{1-\delta^{2}} \psi_{L} .
\end{aligned}
$$

$u_{L+2}-\delta u_{L+1}=-\delta u_{L+1}$

Thus, $\psi_{L} \leq 0 \leq \psi_{L-1}$.

3b) For $n \leq L-2$,

$$
\begin{aligned}
v_{n}-\delta v_{n+1} & =\delta^{n}\left[v_{0}+n\left(1-\delta^{2}\right) u_{0}\right]-\delta^{n+2}\left[v_{0}+(n+1)\left(1-\delta^{2}\right) u_{0}\right] \\
& =\delta^{n}\left(1-\delta^{2}\right)\left[v_{0}+n u_{0}-\delta^{2}(n+1) u_{0}\right] \\
& =\frac{1-\delta^{2}}{\delta}\left[\bar{v}_{n+1}-\underline{u}_{n+1}\right] \geq 0 .
\end{aligned}
$$

For $n=L-1$,

$$
\begin{aligned}
v_{L-1}-\delta v_{L} & =\bar{v}_{L-1}-\frac{\delta^{2}}{1-\delta^{2}} \psi_{L-1} \\
& =\frac{\delta^{L-1}}{1-\delta^{2}}\left(\left(1-\delta^{2}\right)\left[v_{0}+(L-1)\left(1-\delta^{2}\right) u_{0}\right]-\delta^{2}\left[v_{0}+u_{0}+(L-1)\left(1-\delta^{2}\right) u_{0}\right]\right) \\
& =\frac{\delta^{L-1}}{1-\delta^{2}}\left(\left(1-\delta^{2}\right)\left[v_{0}+u_{0}+(L-2)\left(1-\delta^{2}\right) u_{0}\right]-\delta^{2}\left[v_{0}+u_{0}+L\left(1-\delta^{2}\right) u_{0}\right]\right) \\
& =\frac{1}{1-\delta^{2}}\left(\delta\left(1-\delta^{2}\right) \psi_{L-2}-\delta \psi_{L}\right) \\
& =\frac{\delta}{1-\delta^{2}}\left(\left(1-\delta^{2}\right) \psi_{L-2}-\psi_{L}\right)
\end{aligned}
$$

For $n=L, v_{L^{-}} \delta v_{L+1}=v_{L} \geq 0$.

Thus, if $L>0, \psi_{L} \leq 0 \leq \psi_{L-1}$. For $L=0, v_{1}=v_{2}=u_{2}=0$.

$$
u_{1}=v_{1}+\delta u_{2}-\delta v_{0}=-\delta v_{0}<0 \text {. }
$$

The constraint $0<u_{1}-\delta u_{0}=-\delta\left[u_{0}+v_{0}\right]=-\delta \psi_{0}$, which gives $\psi_{0}<0$. 
Thus, in the case of a sequence of zeros, $L$ is the first point $n$ for which $\psi_{n}<0$, which may be 0 . The right side, at $H$, is symmetric.

The Case 1 equilibrium arise provided $L<n_{L}-1, H>n_{R}+1$, i.e. $n^{*}$ exists. $L$ is the largest integer less than, or equal to, $n^{*}$, and thus there is at most one such equilibrium. Neither case 2 nor case 3 arise when $\psi_{n^{*}}>0$, so in this event, the equilibrium is unique. This demonstrates part (a) of the theorem.

Case 2 equilibria look, in terms of the theorem, like case 3 equilibria and thus may be lumped together. (In fact, case 2 equilibria are case 3 equilibria with a degenerate run of zeros). These equilibria are determined by the conditions $\psi_{L} \leq 0 \leq \psi_{L-1}$ and $\phi_{H+1} \geq 0 \geq \phi_{H}$ and thus there is only one such equilibrium. It is unique when the case 1 equilibrium can't exist. This demonstrates parts (b) and (c).

Three years later, this completes the proof. Q.E.D.

\section{Proof of Lemma 3:}

$$
\text { For } n<L, G_{n}(x)=\frac{x}{\delta\left(u_{n+1}-u_{n-1}\right)}=\frac{x}{x} .
$$

Thus,

$$
\begin{gathered}
p_{n}=\int_{0}^{\bar{x}} F_{n}(y) G_{n}^{\prime}(y) d y=\frac{v_{n}-\delta v_{n+1}}{\delta\left(v_{n-1}-v_{n+1}\right)}+\int_{0}^{\bar{x}} \frac{x}{\delta\left(v_{n-1}-v_{n+1}\right)} \frac{1}{\bar{x}} d x=\frac{v_{n}-\delta v_{n+1}+\bar{x} / 2}{\delta\left(v_{n-1}-v_{n+1}\right)} \\
=\frac{v_{n}-\delta v_{n+1}+\left(\delta v_{n-1}-v_{n}\right) / 2}{\delta\left(v_{n-1}-v_{n+1}\right)}=1-\frac{1}{2}\left(\frac{\delta v_{n-1}-v_{n}}{\delta\left(v_{n-1}-v_{n+1}\right)}\right)
\end{gathered}
$$

Substitution of (10) and (11) completes the proof. The case $n>H$ is similar.

Q.E.D.

Proof of Theorem 2: (a) follows immediately from the fact that, for $n<L$, the sum of utilities is $\psi_{n}$, which is decreasing up to $L$; then the sum of utilities is $\phi_{n}$, which is increasing. (b) Maximum effort for $n<L$ is $\bar{x}=\delta u_{n+1}-u_{n}=\left(\delta^{2}-1\right) u_{0} \delta^{n}$ and a routine calculation shows that this is decreasing; the other case is similar. Average effort follows from the observation that the distribution of effort is uniform. The proof of Lemma 3 makes it obvious that $p_{n}>1 / 2$ for $n<L$. Part (d) follows immediately from (18).

\section{Proof of Lemma 4:}

Rewrite (19) to give $q_{n+1}-q_{n}=\frac{p_{n}}{1-p_{n}}\left(q_{n}-q_{n-1}\right)$.

This formula inducts to give $q_{n+1}-q_{n}=q_{1} \prod_{j=1}^{n} \frac{p_{n}}{1-p_{n}}$, and from this, $q_{n+1}=q_{1} \sum_{k=0}^{n}\left(\prod_{j=1}^{k} \frac{p_{n}}{1-p_{n}}\right)$ Since $q_{N}=1$, we obtain (21).

Rewrite (20) to give $\Delta_{n+1}-\Delta_{n}=\frac{p_{n}}{1-p_{n}}\left(\Delta_{n}-\Delta_{n-1}\right)-\frac{1}{1-p_{n}}$. A similar procedure to the calculation of $q_{n}$ gives $\Delta_{n+1}-\Delta_{n}=\Delta_{1} \prod_{j=1}^{n} \frac{p_{j}}{1-p_{j}}-\sum_{j=1}^{n}\left(\frac{1}{1-p_{j}} \prod_{k=j+1}^{n} \frac{p_{k}}{1-p_{k}}\right)$ 
$\Delta_{n+1}=\Delta_{1} \sum_{m=0}^{n}\left(\prod_{j=1}^{m} \frac{p_{j}}{1-p_{j}}\right)-\sum_{m=1}^{n}\left(\sum_{j=1}^{m} \frac{1}{1-p_{j}} \prod_{k=j+1}^{m} \frac{p_{k}}{1-p_{k}}\right)$. The derivation of (22) is completed by

substituting $\Delta_{N}=0$ to solve for $\Delta_{1}$.

Q.E.D.

\section{Limiting Solution}

For $n=\lambda N, \delta^{n}=\left(e^{-\beta}\right)^{\lambda}=e^{-\lambda \beta}$.

$\lim n\left(1-\delta^{2}\right)=\lim \lambda N\left(1-\delta^{2}\right)=\lambda \lim \frac{-\beta\left(1-\delta^{2}\right)}{\log (\delta)}=2 \lambda \beta$.

When combined with (16) and (17), these give (24) and (25). The remark follows immediately from the three parts of Theorem 1. (Footnote (13) verifies that $n_{L}$ and $n_{R}$ occur at the minima of $\psi$ and $\phi$, for the discrete case (and consequently for the limit).

The maximum effort, $\bar{x}$, satisfies, for $\lambda<\lambda^{*}$.

$N \bar{x}=N \delta^{n}\left(\delta^{2}-1\right) u_{0} \rightarrow-2 \lambda \beta u_{0} e^{-\lambda \beta}$.

The limits of $q_{n}$ and $\Delta_{n}$ require a bit of machinery. The first observation is that for a bounded series $z_{n}=z(n / N)$,

$$
\frac{\sum_{n=0}^{N-1} z_{n}\left(\prod_{j=1}^{n} \frac{p_{j}}{1-p_{j}}\right)}{\sum_{n=0}^{N-1}\left(\prod_{j=1}^{n} \frac{p_{j}}{1-p_{j}}\right)} \rightarrow z\left(\lambda^{*}\right) .
$$

(A*) arises because $p_{n}>1 / 2$ if, and only if, $n / N<\lambda^{*}$. Thus, as $N$ gets large, an increasing weight of the probability mass in the expectation embodied in (A8) is placed near $n=\lambda^{*} N$. (27) follows immediately from (21). The second observation is that, if $z_{n}=z(n / N)<1-\varepsilon$, for some $\varepsilon>0$,

$$
\sum_{m=0}^{n}\left(\prod_{j=1}^{m} z_{k}\right) \rightarrow \frac{1}{1-z_{0}}
$$

(A9) follows from noting that, as $N$ gets large, all of the relevant terms in the summation are very nearly powers of $z_{0}$. Formally, let $z_{a}$ be the maximum of $z$ over $[0, a]$, and $z_{\max }$ the maximum over $[0,1]$. Then

$$
\sum_{m=0}^{n}\left(\prod_{j=1}^{m} z_{k}\right)=\sum_{m=0}^{a N}\left(\prod_{j=1}^{m} z_{k}\right)+\sum_{m=0}^{\lambda N}\left(\prod_{j=1}^{m} z_{k}\right) \leq \frac{1-z_{a}^{a N+1}}{1-z_{a}}+\frac{z_{a}^{a N+1}}{1-z_{\max }} \rightarrow \frac{1}{1-z_{a}}
$$

Sending $a$ to 0 completes the upper bound; the lower bound is analogous (using minima). 
Note, from (22) and (A8), that $(n *=\lambda * N)$ :

$$
\Delta_{1}=\frac{\sum_{m=0}^{N-1} \sum_{j=1}^{m} \frac{1}{1-p_{j}} \prod_{k=j+1}^{m} \frac{p_{j}}{1-p_{j}}}{\sum_{n=0}^{N-1} \prod_{j=1}^{n} \frac{p_{j}}{1-p_{j}}}=\frac{\sum_{m=0}^{N-1} \prod_{j=1}^{n} \frac{p_{j}}{1-p_{j}} \sum_{j=1}^{m} \frac{1}{1-p_{j}} \prod_{k=j+1}^{m} \frac{1-p_{j}}{p_{j}}}{\sum_{n=0}^{N-1} \prod_{j=1}^{n} \frac{p_{j}}{1-p_{j}}} \approx \sum_{j=1}^{n^{*}} \frac{1}{1-p_{j}} \prod_{k=j+1}^{m} \frac{1-p_{j}}{p_{j}}
$$

Thus, for $n<\lambda N$

$$
\begin{aligned}
& \Delta_{n}=\Delta_{1} \sum_{m=0}^{n-1}\left(\prod_{j=1}^{m} \frac{p_{j}}{1-p_{j}}\right)-\sum_{m=0}^{n-1} \sum_{j=1}^{m}\left(\frac{1}{1-p_{j}} \prod_{k=j+1}^{m} \frac{p_{k}}{1-p_{k}}\right) \\
&= \sum_{m=0}^{n-1}\left[\prod_{j=1}^{m} \frac{p_{j}}{1-p_{j}}\left(\Delta_{1}-\sum_{j=1}^{m} \frac{1}{1-p_{j}} \prod_{k=1}^{j} \frac{1-p_{k}}{p_{k}}\right)\right] \\
& \approx \sum_{m=0}^{n-1}\left[\prod_{j=1}^{m} \frac{p_{j}}{1-p_{j}}\left(\sum_{j=1}^{n^{*}}\left(\frac{1}{1-p_{j}} \prod_{k=1}^{j} \frac{1-p_{j}}{p_{j}}\right)-\sum_{j=1}^{m}\left(\frac{1}{1-p_{j}} \prod_{k=1}^{j} \frac{1-p_{k}}{p_{k}}\right)\right)\right] \\
&=\sum_{m=0}^{n-1}\left[\prod_{j=1}^{m} \frac{p_{j}}{1-p_{j}}\left(\sum_{j=m+1}^{n^{*}} \frac{1}{1-p_{j}} \prod_{k=1}^{j} \frac{1-p_{j}}{p_{j}}\right)\right] \\
&=\sum_{m=0}^{n-1}\left(\sum_{j=m+1}^{n^{*}} \frac{1}{1-p_{j}} \prod_{k=1}^{j} \frac{1-p_{j}}{p_{j}}\right)=\sum_{m=0}^{n-1}\left(\sum_{j=m+1}^{n^{*}} \frac{1}{p_{j}} \prod_{k=1}^{j-1} \frac{1-p_{j}}{p_{j}}\right) \\
& \approx \sum_{m=0}^{n-1} \frac{1}{p_{m}} \frac{1}{1-\frac{1-p_{m}}{p_{m}}} \approx N \int_{0}^{n} \frac{1}{2 p(x)-1} d x . \\
&
\end{aligned}
$$

The case of $n>n *$ is symmetric; this completes the derivation of (28).

\section{Patient Players}

For $u_{0}+v_{0}=u_{N}+v_{N}$, rewrite $\psi_{n}=\phi_{n}$ as

$$
\frac{\boldsymbol{\delta}^{n}-\boldsymbol{\delta}^{N-n}}{1-\boldsymbol{\delta}^{2}}\left(u_{0}+v_{0}\right)=-\boldsymbol{\delta}^{n} n u_{0}+\boldsymbol{\delta}^{N-n}(N-n) v_{N}
$$

Using L'Hopital, we obtain

$$
\frac{n-(N-n)}{-2}\left(u_{0}+v_{0}\right)=-n u_{0}+(N-n) v_{N}
$$

Solving for $n$, using $u_{0}+v_{0}=u_{N}+v_{N}$, gives $\frac{n^{*}}{N}=\frac{u_{N}-v_{N}}{u_{N}-v_{N}+v_{0}-u_{0}}$. 